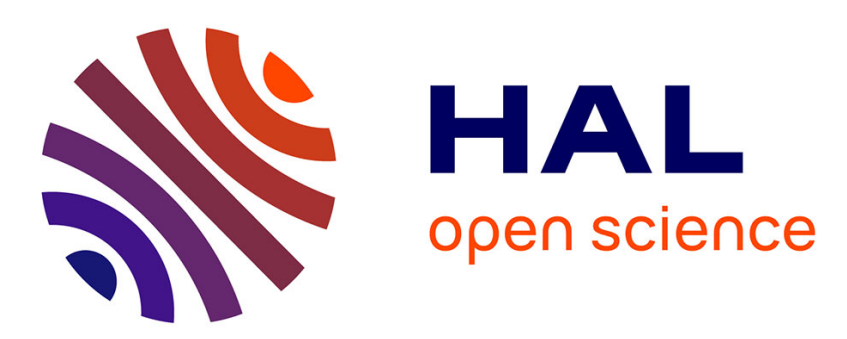

\title{
Progress in analytical methods to predict and control azimuthal combustion instability modes in annular chambers
}

\author{
Michaël Bauerheim, Franck Nicoud, Thierry Poinsot
}

\section{To cite this version:}

Michaël Bauerheim, Franck Nicoud, Thierry Poinsot. Progress in analytical methods to predict and control azimuthal combustion instability modes in annular chambers. Physics of Fluids, 2016, 28 (2), pp.021303. 10.1063/1.4940039 . hal-01269821

\section{HAL Id: hal-01269821 \\ https://hal.science/hal-01269821}

Submitted on 5 Feb 2016

HAL is a multi-disciplinary open access archive for the deposit and dissemination of scientific research documents, whether they are published or not. The documents may come from teaching and research institutions in France or abroad, or from public or private research centers.
L'archive ouverte pluridisciplinaire HAL, est destinée au dépôt et à la diffusion de documents scientifiques de niveau recherche, publiés ou non, émanant des établissements d'enseignement et de recherche français ou étrangers, des laboratoires publics ou privés. 


\section{OATAO \\ Open Archive Toulouse Archive Ouverte}

\section{Open Archive TOULOUSE Archive Ouverte (OATAO)}

OATAO is an open access repository that collects the work of Toulouse researchers and makes it freely available over the web where possible.

This is an author-deposited version published in : http://oatao.univ-toulouse.fr/ Eprints ID : 15121

To link to this article : DOI:10.1063/1.4940039

URL : http://dx.doi.org/10.1063/1.4940039

\section{To cite this version :}

Bauerheim, Michaël and Nicoud, Franck and Poinsot, Thierry Progress in analytical methods to predict and control azimuthal combustion instability modes in annular chambers. (2016) Physics of Fluids, vol. 28 ( $\left.\mathrm{n}^{\circ} 2\right)$. pp. 021303. ISSN 1070-6631

Any correspondence concerning this service should be sent to the repository administrator: staff-oatao@listes-diff.inp-toulouse.fr 


\title{
Progress in analytical methods to predict and control azimuthal combustion instability modes in annular chambers
}

\author{
M. Bauerheim, ${ }^{1}$ F. Nicoud, ${ }^{2}$ and T. Poinsot ${ }^{3, a)}$ \\ ${ }^{1}$ Cerfacs, CFD Team, 42 Avenue Gaspard Coriolis, 31057 Toulouse, France \\ ${ }^{2}$ Université de Montpellier. IMAG, UMR CNRS 5149, Montpellier, France \\ ${ }^{3}$ IMF Toulouse, INP de Toulouse and CNRS, 31400 Toulouse, France
}

\begin{abstract}
Longitudinal low-frequency thermoacoustic unstable modes in combustion chambers have been intensively studied experimentally, numerically, and theoretically, leading to significant progress in both understanding and controlling these acoustic modes. However, modern annular gas turbines may also exhibit azimuthal modes, which are much less studied and feature specific mode structures and dynamic behaviors, leading to more complex situations. Moreover, dealing with 10-20 burners mounted in the same chamber limits the use of high fidelity simulations or annular experiments to investigate these modes because of their complexity and costs. Consequently, for such circumferential acoustic modes, theoretical tools have been developed to uncover underlying phenomena controlling their stability, nature, and dynamics. This review presents recent progress in this field. First, Galerkin and network models are described with their pros and cons in both the temporal and frequency framework. Then, key features of such acoustic modes are unveiled, focusing on their specificities such as symmetry breaking, non-linear modal coupling, forcing by turbulence. Finally, recent works on uncertainty quantifications, guided by theoretical studies and applied to annular combustors, are presented. The objective is to provide a global view of theoretical research on azimuthal modes to highlight their complexities and potential. (C) 2016 AIP Publishing LLC. [http://dx.doi.org/10.1063/1.4940039]
\end{abstract}

\section{INTRODUCTION}

\section{A. Combustion instability}

Large power densities in gas turbines can be accompanied by fluctuations leading to potential problems known as combustion instabilities. ${ }^{1,2}$ These oscillations are due to a coupling between the flame and the acoustics, creating high pressure and heat release oscillations in the chamber, which may destroy the whole propulsion system. ${ }^{3,4}$ Therefore, over the last sixty years, combustion instabilities have constantly been a key issue for rocket engines, and more recently for gas turbines used in aeronautics and power generation. A huge research effort ${ }^{1,2,5}$ has been dedicated to the investigation of coupling mechanisms and the control of unstable modes in high-performance engines. ${ }^{1,6,7}$

An underlying challenge in modeling combustion instabilities is the difficulty to perform full-scale experiments, or at least to carry out detailed measurements in the chamber due to the extreme conditions and the complex physics involved ${ }^{8-11}$ Moreover, the necessity to perform experiments with inlet and outlet impedances matching real engine conditions makes direct comparison

Note: This paper is based on an invited plenary lecture given by T. Poinsot at the 67th Annual Meeting of the American Physical Society, which was held 23-25 November 2014 in San Fransisco, CA, USA.

a)Poinsot@imft.fr 


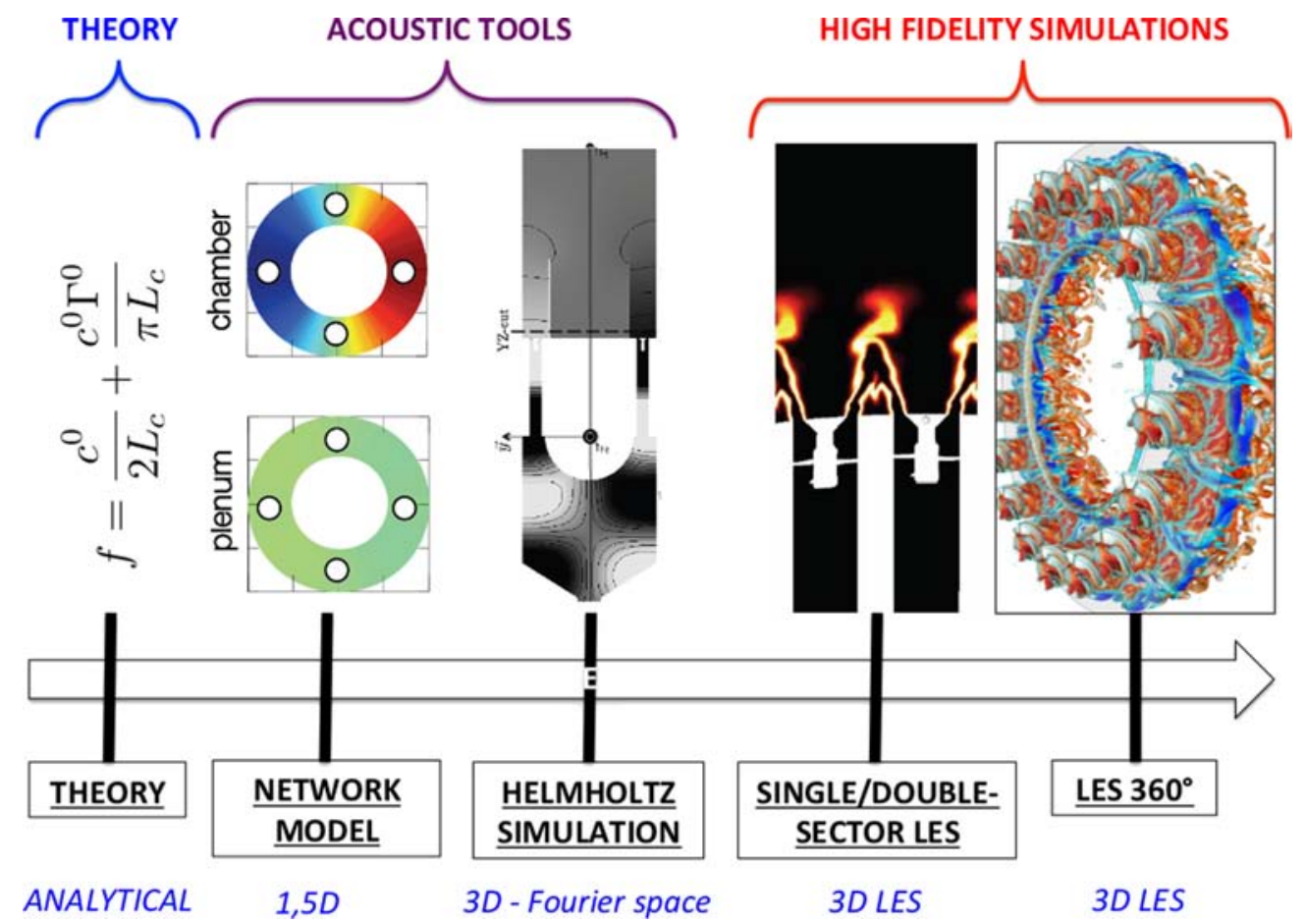

FIG. 1. Overview of methods and tools used to investigate and predict combustion instabilities, from analytical theories (left) to full $360^{\circ}$ large Eddy simulations (right).

of laboratory results and real engine data difficult. Using simulation (Fig. 1, right) has become an attractive additional method: thanks to the increase of the computational power, numerical simulations on massively parallel computers can replicate the complex mechanisms involved in combustion instabilities in full-scale geometries. ${ }^{12-14}$ Low-order models (Fig. 1, middle) and even theory (Fig. 1, left) on simple geometries ${ }^{15-19}$ are also developed to guide Large Eddy Simulation (LES) and experiments. This is a crucial step to improve the understanding of these phenomena. Nevertheless, the usefulness of such analytical approaches is often questioned but rarely addressed. This paper presents recent progress in analytical methods for combustion instabilities and shows how they complement simulation and experiment to investigate phenomena leading to combustion instabilities, predict unstable modes, and control them if they appear.

\section{B. The specific case of azimuthal modes}

A specific case where combustion instabilities raise multiple fundamental questions corresponds to the annular chambers of many gas turbines: in these configurations, instabilities are often caused by azimuthal modes (Figs. 2 and 3) propagating along the azimuthal direction $\vec{e}_{\theta}$ and not only in the longitudinal direction $\vec{e}_{z} \cdot{ }^{2,4,20,22}$ Mechanisms leading to azimuthal instabilities are more complex than those encountered in longitudinal configurations. As presented in Fig. 4, which focuses on neighboring burners of an annular rig (Fig. 2, right), the azimuthal mode acts like a clock which modulates the axial mass flow rate in the burners (Fig. 4(a)) but also creates a transverse excitation (Fig. 4(b)), especially in burners located at a pressure node. ${ }^{22,23}$ These two mechanisms wrinkle the flame and affect its surface and position: they can promote flame-flame interaction (Fig. 4(c), also known as flame merging) but the effect of this interaction on thermo-acoustic instabilities is not fully understood. ${ }^{24-27}$ Note that the mechanisms presented in Fig. 4 assume that burners are compact with respect to the acoustic wavelength, an assumption which is usually valid for azimuthal modes in annular combustors: $\lambda \simeq 2 L_{c} \gg a$ where $\lambda$ is the azimuthal wavelength, $2 L_{c}=2 \pi R_{c}$ is the perimeter of the annular chamber, and $a=\sqrt{4 S_{i} / \pi}$ is the burner diameter based on the burner cross-section $S_{i}$. Note that recently, high-frequency thermoacoustic instabilities in 


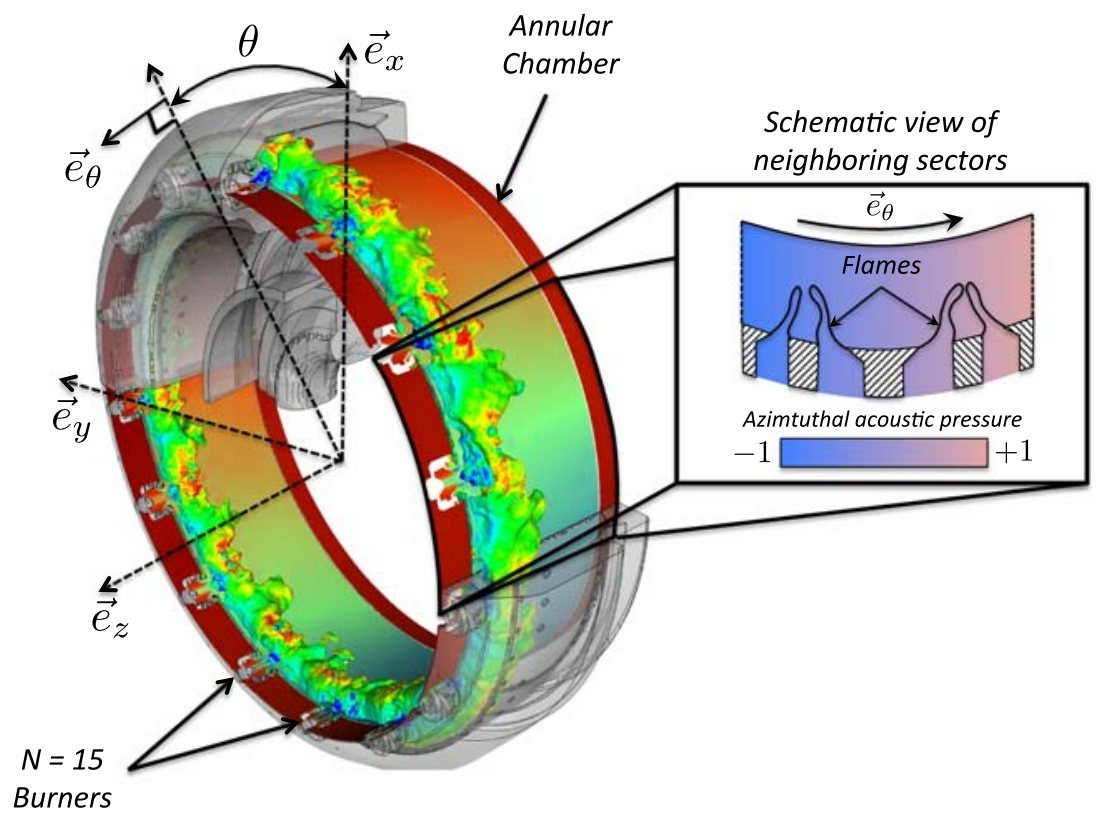

FIG. 2. 3D view (left) and schematic view (right) of an azimuthal combustion instability (pressure fluctuations along the azimuthal direction $\vec{e}_{\theta}$ ) in an annular engine (left) and zoom on two neighboring burners (right).

annular combustors have been observed experimentally and numerically for which this assumption does not hold. ${ }^{28,29}$

The situation becomes more complex when the annular chamber is coupled to an annular plenum (Fig. 3). Since they have different radii $\left(R_{p} \neq R_{c}\right)$ and mean sound speed $\left(c_{u}^{0} \neq c^{0}\right)$, thus different resonant frequencies, azimuthal modes should be affected by only one cavity (plenum or chamber) at a time: the two cavities are "decoupled." ${ }^{30}$ However, experiments and simulations ${ }^{30-33}$

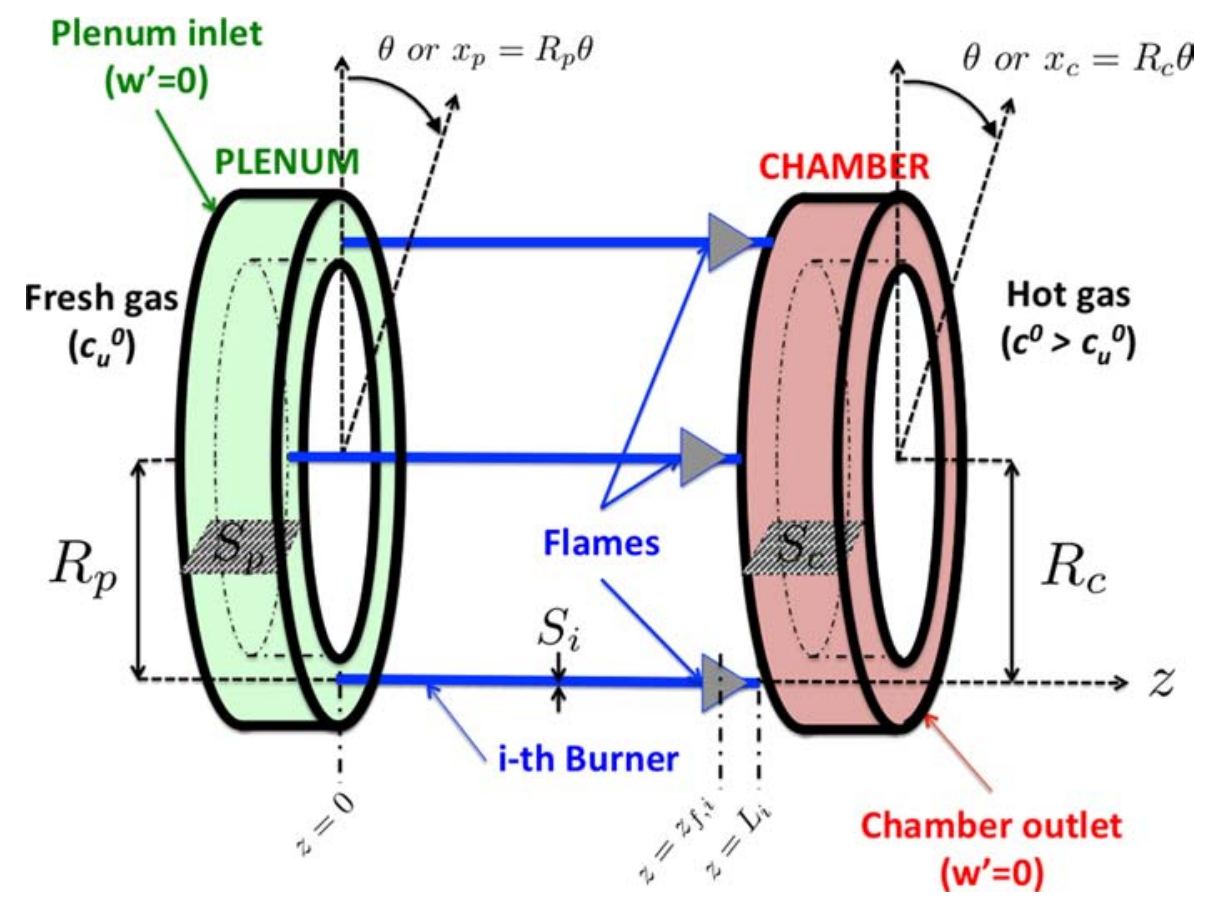

FIG. 3. Annular chamber (right) connected to $N$ burners fed by a common annular plenum (left). 


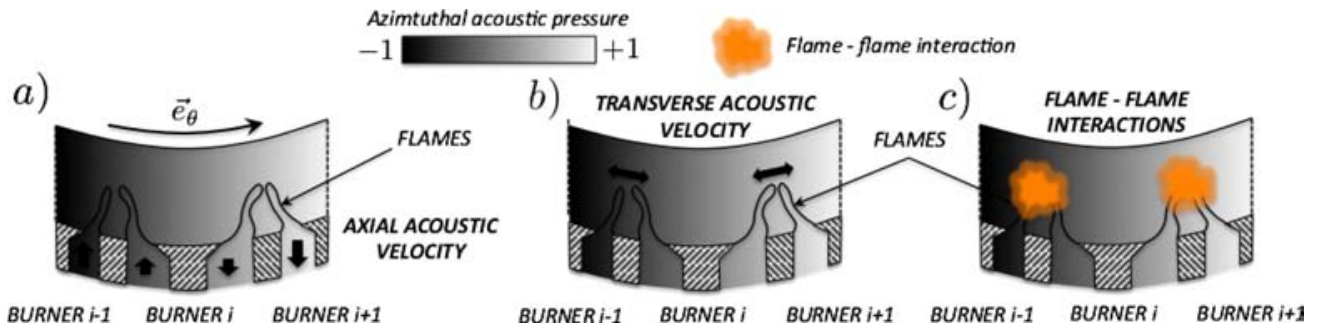

FIG. 4. Azimuthal mechanisms driving combustion instabilities: the azimuthal mode acts like a clock modulating the axial mass flow rate in the burners (a) but also creates a transverse excitation (b). Both mechanisms can also generate flame-flame interaction (c), whose effect on thermo-acoustic instabilities is not yet understood.

show that azimuthal modes can develop simultaneously in both cavities. This observation raises questions on how annular cavities can couple and which cavity drives the azimuthal mode. It is a crucial information to control azimuthal modes in industrial gas turbines, which usually contain multiple cavities.

The structure of azimuthal modes, either in the annular plenum or chamber, has been also a continuous topic of discussion. The work performed at Siemens ${ }^{34}$ proved that multiple modal structures could coexist in a real gas turbine: spinning, standing, or even mixed modes can appear successively, and instabilities can switch from one structure to another. ${ }^{35,36}$ Structures of spinning, standing and mixed modes are represented in Fig. 5: each azimuthal mode is composed of two components, e.g., clockwise/counter-clockwise. Depending on the configuration, these two components can be similar (i.e., same frequency and growth rate, they are called "degenerate" ${ }^{37}$ ) or different (i.e., with different frequency and stability criterion, they are called "non-degenerate" ${ }^{37}$ ). The final mode structure is determined by the combination of these two components, which is still an open topic today. A first LES attempt to understand this peculiar phenomenon was carried out by Wolf et al., ${ }^{12}$ who showed that swirlers induce a mean azimuthal velocity which can modify the flame-flame or flame-acoustics interactions and thus influence the spinning or standing modes occurrence, as also observed in the Dawson's experiment. ${ }^{38,39}$ Schuermans et al. have also argued in 2006 that at high pressure oscillation levels, only turning modes would survive because

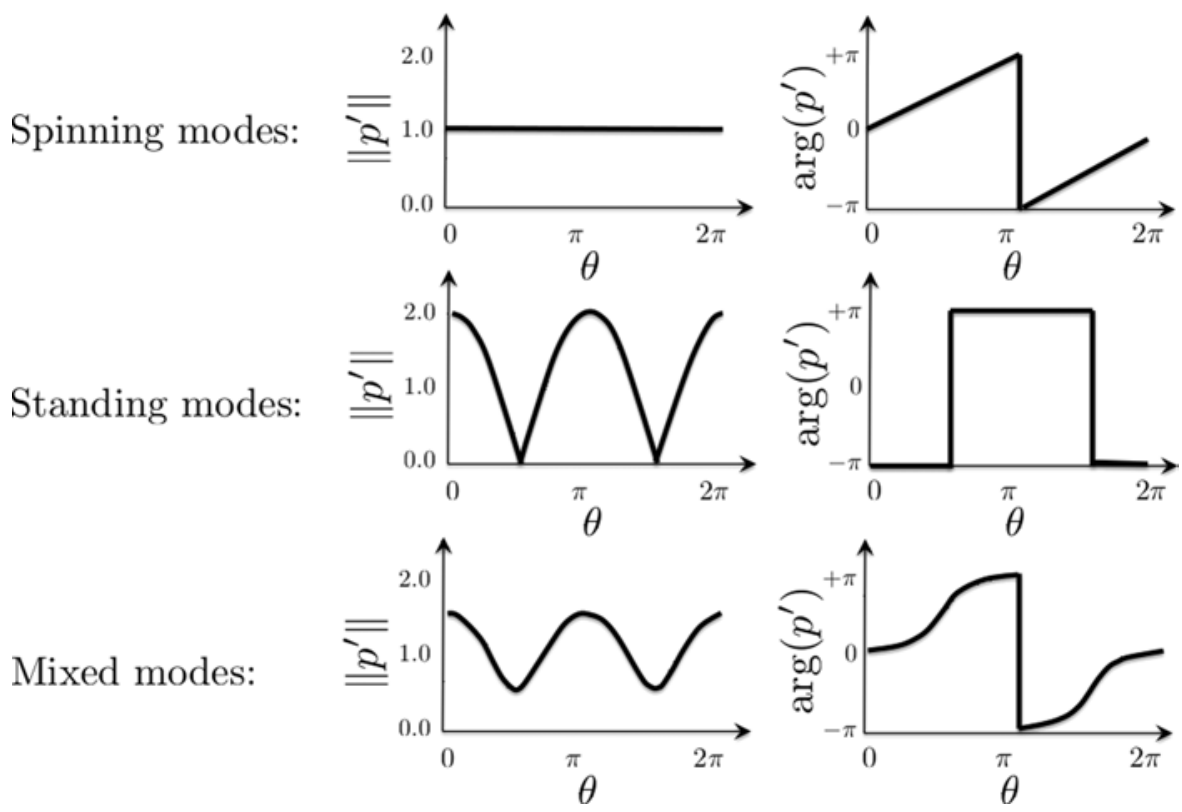

FIG. 5. Structure of spinning (top), standing (middle), and mixed (bottom) modes determined by the modulus (left) and phase (right) of the complex pressure oscillations plotted as a function of the azimuthal position $\theta$. 
of non-linear effects. ${ }^{35}$ Sensiau et al. and Noiray et al. demonstrated that turning modes would appear only in purely axisymmetric cases, standing or mixed modes occurring otherwise ${ }^{40-42}$ However, the effect of the modes structure (standing, spinning) on the flame-acoustics interactions and therefore on instabilities is still an open question. For instance, the new mode pattern ("slanted mode") observed by the EM2C group in their academic annular combustor MICCA ${ }^{43}$ calls for novel theoretical developments.

\section{Objectives: Understand, predict, and control azimuthal modes}

The objective of this paper is to investigate the different analytical approaches developed for azimuthal modes and determine the fundamental aspects they can unveil. First, an overview of existing methods is provided in Section II. Then, this paper emphasizes several aspects of combustion instabilities: Section III derives key parameters controlling azimuthal modes in order to classify them. It focuses mainly on coupled/decoupled modes (Section III A), symmetry breaking (Section III B), and control of azimuthal modes, as well as mode dynamics during limit cycles (Section III C). The recent topics of stochastic approaches and uncertainty quantification (UQ) for combustion instabilities are addressed in Sections III D and III E. This last class of methods strongly relies on cost-effective low-order models and theories, which allows uncertainty quantification (UQ) issues to be addressed in combustion instability studies.

\section{OVERVIEW OF ANALYTICAL APPROACHES}

Even if experiments remain absolutely necessary, there has been only a few experimental studies dealing with azimuthal combustors because of their cost and complexity. ${ }^{17,32,38,39,43,44}$ Large Eddy simulations of full annular chambers are also still rare and difficult to post-process, ${ }^{12-14,45,46}$ and therefore low-order models (such as 3D acoustic solvers, not detailed in this paper) and theory constitute essential elements of the research on combustion instabilities. However, theory is often neglected, because developing theory in complex geometry systems is a challenge.

\section{A. A first attempt to understand combustion instability: The Rayleigh criterion}

Since the 1950s, an important research effort has been performed on the underlying phenomena governing the feed-back loop and the flame-sound interaction leading to instabilities. ${ }^{5,47,48}$ The first stability criterion for thermoacoustic instabilities was provided very early by Lord Rayleigh ${ }^{49}$ based on the acoustic energy balance equation, which reads

$$
\int_{\Omega} \frac{\gamma-1}{\gamma p_{0}}\left\langle p^{\prime} \dot{\omega}_{T}{ }^{\prime}\right\rangle d \Omega<\int_{A}\left\langle p^{\prime} \vec{u}^{\prime}\right\rangle \cdot \vec{n} d A,
$$

where $p^{\prime}$ and $\vec{u}^{\prime}$ are the acoustic pressure and velocity, $\dot{\omega}_{T}{ }^{\prime}$ the fluctuating heat release, and $\langle$.$\rangle is$ a temporal averaging. This criterion reveals that the balance between flame-acoustics interactions (LHS) and acoustic fluxes at boundaries acting like damping (RHS) controls the combustion instabilities. Note that in practice, other damping phenomena may occur, for instance, due to the acoustic boundary layer at walls, ${ }^{50}$ acoustics-vorticity conversion, etc. In an annular chamber, the Rayleigh criterion becomes more complicated since every burner can increase or decrease the LHS term of Eq. (1) at a given instant. Unlike longitudinal modes in single burner experiments, azimuthal modes require to analyze the spatial structure of the interaction term $\left\langle p^{\prime} \dot{\omega}_{T}{ }^{\prime}\right\rangle$ as a function of the azimuthal position. Eventually, one may note that the Rayleigh criterion can only be used a posteriori since it requires the knowledge of the mode structure.

Moreover, the source terms $\dot{\omega}_{T}^{\prime}$ due to the flames are usually modeled by a Flame Transfer Function $\left(\mathrm{FTF}^{51}\right)$, or its non-linear equivalent Flame Describing Function $\left(\mathrm{FDF}^{16,52}\right)$, which relates heat release fluctuations $\left({\dot{\omega_{T}}}^{\prime}\right)$ to acoustic fluctuations (the pressure $p^{\prime}$ or the longitudinal velocity $\left.w^{\prime}\right)$ : the spatial structure of the acoustic mode $\left(p^{\prime}\right.$ or $\left.w^{\prime}\right)$ is coupled to the flames (source terms), and thus must be known to determine the stability of the configuration. Numerical tools such as 
$3 D$ Helmholtz solvers ${ }^{19,33}$ have been developed to solve this problem (not detailed in this paper), but theoretical approaches have been also proposed to determine the stability of the system explicitly. Such predictive analytical methods are discussed in Secs. II B and II C.

\section{B. Galerkin vs. network models}

Simple longitudinal configurations were intensively studied in both linear ${ }^{15,31,53-58}$ and nonlinear regimes. ${ }^{16,52,59,60}$ Complex annular configurations were investigated analytically or quasianalytically only recently because of their complexity, mainly based on two different strategies to compute the spatial mode structure $p^{\prime}$ :

- Galerkin projection: This approach usually relies on truncated series, called "Galerkin series," which corresponds to a projection method: the acoustic fluctuations in the presence of flames are searched as a combination of the acoustic modes of the annular cavity without flames, i.e., the eigenfunctions of the homogeneous equation $\eta_{m}(t) \Psi_{m}(\theta),{ }^{1,15,61-64}$

$$
p^{\prime}(\theta, t)=\sum_{m=-\infty}^{\infty} \eta_{m}(t) \Psi_{m}(\theta)
$$

for a pure azimuthal mode in the time-domain, where $\theta$ corresponds to the azimuthal angle. When the "unperturbed" eigenmodes $\Psi_{m}(\theta)$ are orthogonal to each other, i.e., $\int_{0}^{2 \pi} \Psi_{m}(\theta) \Psi_{n}(\theta)$ $d \theta=\delta_{m, n}\left\|\Psi_{m}\right\|^{2}$, it results in a system of non-linearly coupled differential equations for the mode of order $m$, the coupling term being $\frac{\partial \omega_{T^{\prime}}(\theta, t)}{\partial t} \Psi_{m}(\theta)$,

$$
\frac{d^{2} \eta_{m}}{d t^{2}}+\omega_{m}^{2} \eta_{m}=\frac{\gamma-1}{\left\|\Psi_{m}\right\|^{2}} \int_{0}^{2 \pi} \frac{\partial \dot{\omega}_{T}^{\prime}(\theta, t)}{\partial t} \Psi_{m}(\theta) d \theta .
$$

The coupling term, involving the unknown heat-release fluctuations $\dot{\omega}_{T}{ }^{\prime}$, requires a proper flame-model description, which can complexify drastically the analytical resolution of Eq. (3). While simplistic models based on the acoustic pressure like $\dot{\omega}_{T}{ }^{\prime}(t)=n p^{\prime}(t)$ are often used, ${ }^{61}$ delayed-models lead to delayed differential equations for which analytical solutions are not straightforward. ${ }^{63}$ The problem may become no longer tractable when more complex flame models, depending on the frequency or including non-linear saturation effects, are taken into account. Additionally, for complex configurations or boundary conditions, the mode structure $\Psi_{m}(\theta)$ is not trivial and prevents the full analytical resolution of the problem. A semi-analytical method is possible by computing the mode $\Psi_{m}(\theta)$ with a $3 \mathrm{D}$ acoustic solver based on finite-volume or finite-element methods ${ }^{19,32,65,66}$ (not detailed in this paper). Analytical results for $\Psi_{m}$ can be obtained in simple annulus (one annular cavity with no burner, (a) in Fig. 6), for instance, where $\Psi_{m}$ can be approximated by $\Psi_{m}=e^{j m \theta}$. Recently, Bourgouin ${ }^{32}$ proposed a method to study Plenum-Burners-Chamber configuration (PBC) configuration ((c)

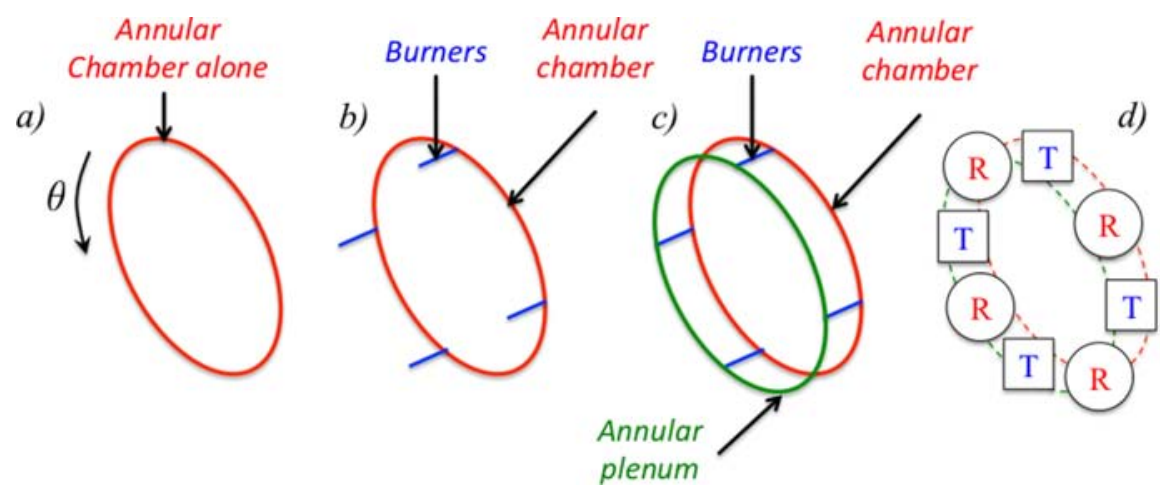

FIG. 6. Examples of annular configurations studied analytically. (a) Simple annulus, (b) One annular cavity coupled with $N$ burners (BC configuration), (c) Two annular cavities coupled with $N$ burners (PBC configuration) and (d) Network representation of the annular combustor with propagation $(\mathrm{R})$ and interaction $(\mathrm{T})$ matrices. 
in Fig. 6) by applying a Galerkin approach on the two annular cavities separately. The annular plenum was then connected to the annular chamber by scattering matrices describing the burners, which link acoustic quantities in the plenum to those in the chamber. Because of the orthogonality assumption, Eq. (3) cannot be obtained when the unperturbed eigenfunctions $\Psi_{m}$ are not orthogonal. In this case, the Galerkin method can still be used, but yields a system of coupled equations, which usually prevent its full analytical resolution. However, it can be solved numerically to study non-normality and non-linear triggering, ${ }^{60}$ for instance, due to non-trivial boundary conditions. ${ }^{19}$

- Network model: Another approach is to solve the thermoacoustic problem using a quasi-1D description of the geometry, ${ }^{30,65,67-71}$ one of this approach being the ATACAMAC network model..$^{30,70-72}$ A network model can be constructed using 1D tubes where acoustic propagation takes place only along the duct axis (characterized by a propagation matrix $R_{i}(\omega)$ ). Between the tubes, acoustic jump conditions ${ }^{15,73}$ lead to an interaction matrix $T_{i}\left(\Gamma_{i}, \omega\right)$, which describes the interaction between the acoustic mode and the flames ((d) in Fig. 6). $\Gamma_{i}$ is called the "coupling parameter" and is associated with the intensity of the coupling between the two tubes and the flames, i.e., the equivalent admittance of the burner/chamber junction. This approach was first developed for longitudinal cases $^{31}$ and then adapted to annular configurations using the Annular Network Reduction (ANR) method. ${ }^{70,71}$ It yields an analytical dispersion relation containing small matrices (typically $2 C \times 2 C$, where $C$ is the number of annular cavities in the configuration),

$$
\operatorname{det}\left(\prod_{i=N}^{1} R_{i}(\omega) T_{i}(\omega)-I_{d}\right)=0,
$$

where $I_{d}$ is the identity matrix. This analytical dispersion relation can be solved, either fully analytically or numerically using appropriate root finding methods. While explicit analytical solutions are usually restrained to pure azimuthal modes, the second quasi-analytical method allows the investigation of mixed modes. However, since this review focuses on explicit solutions for azimuthal modes, numerical tools based on analytical network approaches are not detailed hereafter. For both methods, the mode structure is not assumed a priori, therefore this approach can be applied on configurations with burners and multiple annular cavities, even when the rotational symmetry is broken (orthogonality is not required).

Consequently, Galerkin and network approaches are widely used to compute acoustic modes in simplified systems. Theoretical studies to adapt these two methods to more realistic annular combustors (PBC configurations, complex boundary conditions, non-null mean flows, etc.) were performed only recently ${ }^{30,32,71}$ and are still in progress. To summarize, on one hand, network models are designed to handle complex geometries by connecting 1D tubes. However, because of its matrix formulation, it usually remains in the linear regime and computes modes separately. On the other hand, Galerkin methods were designed for simple geometries, and adapt them to more complex cases is not straightforward. Compared with the matrix formulation of network models, Galerkin methods usually provide a system of differential equations to be solved, which allows the study of non-linear regimes and non-linear modal coupling, shown in Section III C to control the azimuthal mode dynamics. Note also that these two approaches can be combined to construct more complex quasi-analytical tools, giving a numerical approximation of the stability or dynamics of azimuthal modes instead of explicit expressions as presented in this review: for instance, a network model can be built by connecting subsystems obtained by a Galerkin expansion.

\section{Time vs. frequency-domain analysis}

The previous Galerkin and network approaches are used to represent the modal structure in the annular configuration (spatial representation). The equation governing acoustics in the system (Eq. (3) or (4)) is then solved analytically, either in the time or frequency domain: 
- Time-domain methods: The natural approach is to consider the acoustic fluctuations varying in time. In particular, a time-dependent flame model can be chosen to close the problem. For instance, when choosing a delayed linear model, similar to the so-called $n-\tau$ model ${ }^{74}$ but based on the acoustic pressure ${\dot{\omega_{T}}}^{\prime}(\theta, t)=n p^{\prime}(\theta, t-\tau),{ }^{61}$ the RHS term of Eq. (3) can be computed $\left(R H S=n(\gamma-1) \frac{d \eta_{m}(t-\tau)}{d t}\right)$ leading to a Delayed Differential Equation. On one hand, this methodology allows the analytical resolution of the problem in the temporal framework and simple non-linear effects can be easily introduced. ${ }^{61,62,64}$ However, dealing with more generic flame responses to acoustics, for instance frequency-dependent flame models, is not straightforward. The time-domain approach allows also to determine the mode dynamics of azimuthal modes using classical results of the dynamical systems theory (Section III C) ${ }^{61,64}$ Nevertheless, the complexity unveiled in simple longitudinal configurations by such time-domain approaches (non-linear triggering, non-linear bifurcations, quasiperiodic oscillations, chaos, etc. $)^{60,75}$ is still under investigation for azimuthal modes. On the other hand, only simple boundary conditions are usually accounted for, since complex impedances remain difficult to prescribe in a temporal framework.

- Frequency-domain analysis: Another approach is to solve the thermoacoustic problem in the frequency domain. ${ }^{30,65,67-71}$ The acoustic fluctuations are assumed to be harmonic in time, which allows a Fourier representation of the complex acoustic pressure,

$$
\tilde{p}(\theta, t)=\hat{p}(\theta, \omega) e^{-j \omega t},
$$

where the acoustic pressure $p^{\prime}$ is linked to the complex pressure $\tilde{p}$ by the expression $p^{\prime}$ $=\operatorname{Re}(\tilde{p})$ and $\omega$ is the complex angular frequency. This formalism allows a simple treatment of time-derivatives, leading to an algebraic dispersion relation (e.g., Eq. (4)). Compared with the temporal framework, the unknowns are now the complex angular frequencies $\omega=\omega_{m}$ which are searched as roots of the analytical dispersion relation. In particular, the stability of the $m$ th acoustic mode is given by the imaginary part of the complex angular frequency: if $\operatorname{Im}\left(\omega_{m}\right)>0$ the mode is unstable, and if $\operatorname{Im}\left(\omega_{m}\right)<0$ the mode is stable. The dispersion relation in the frequency domain can be solved, either fully analytically (a Taylor expansion is used to search the complex angular frequency as $\omega_{m}=\omega_{m}^{0}+\delta \omega_{m}$, where $\left\|\delta \omega_{m}\right\| \ll \omega_{m}^{0}$ and $\omega_{m}^{0}$ is the frequency of the acoustic mode without burners or flames) or numerically using appropriate root finding methods. This approach is well suited to account for complex boundary conditions through impedances, but non-linear effects and mode dynamics cannot be obtained directly. The introduction of the FDF formalism in frequency-domain tools to include saturation effects and predict limit-cycles, as for longitudinal modes using a $3 D$ Helmholtz solver, ${ }^{76}$ is still an open topic for azimuthal modes in annular configurations.

Table I provides a summary of the pros $(\checkmark)$ and cons $(\times)$ of these two methods for full analytical resolutions. Note that both time and frequency approaches can also be solved numerically to limit their constraints. Since the two methods allow linear predictions this category is not shown in Table I. They can also both include non-linear flame responses, such as saturation effects. However, only the time-domain methods are able to retrieve the non-linear coupling between modes and their dynamics. In contrast, frequency-domain approaches can deal with complex boundary conditions (non-trivial impedances are straightforward to implement in the frequency-domain ${ }^{19}$ ).

TABLE I. Pros $(\checkmark)$ and cons $(\times)$ of time-domain and frequency-domain analysis to predict analytically the stability and the structure of azimuthal acoustic modes.

\begin{tabular}{lcc}
\hline \hline Features & Time-domain & Frequency-domain \\
\hline Non-linear flame response & $\checkmark$ & $\times$ \\
Non-linear modal coupling & $\checkmark$ & $\times$ \\
Complex BCs & $\times$ & $\checkmark$ \\
Complex flame models $^{\mathrm{a}}$ & $\times$ & $\checkmark$ \\
\hline \hline
\end{tabular}

${ }^{\mathrm{a}}$ For instance, frequency-dependent flame transfer functions. 


\section{KEY FEATURES CONTROLLING AZIMUTHAL MODES STABILITY AND DYNAMICS}

Annular chambers of real gas turbines are usually characterized by numerous modes in the low frequency range: ${ }^{19,33}$ typically $10-30$ modes can be identified in large scale industrial combustors between 0 and $300 \mathrm{~Hz}$. One interesting issue is therefore to classify them, a crucial step before controlling them. For example, both standing and spinning ${ }^{34,36}$ modes (Fig. 5) are observed in industrial configurations, ${ }^{34,61,77}$ but predicting which mode type will appear in practice remains difficult. ${ }^{61-63}$ Other typical categories are "longitudinal vs. azimuthal modes," or "modes involving only one part of the combustor (decoupled modes) vs. modes resonating in the whole system (coupled modes).",33,34,78 Knowing that a predicted mode with a specific nature is driven only by a certain part of the combustor is obviously a key asset before applying any passive control strategy. ${ }^{69,79}$

\section{A. Coupled vs. decoupled modes}

The classification of modes in annular combustors is a challenging task. Even if $3 D$ acoustic solvers can determine whether a mode is present in a certain part of the combustor or not, they cannot help understanding the basic mechanisms leading to the growth of these modes. ${ }^{33,79}$ For instance, for combustion chambers including an annular plenum coupled with an annular chamber, theory is useful to understand how azimuthal modes in the plenum and in the chamber can interact or live independently.

These issues can be investigated on an annular chamber (subscript $c$ ) connected to $N$ burners fed by a common annular plenum (subscript $p$ ), called a "PBC configuration" ((c) in Fig. 6), using a network model. ${ }^{30}$ The period of the first azimuthal mode of the chamber without flames is $\tau_{c}^{0}=2 L_{c} / c^{0}$ and its frequency $f_{c}^{0}=c^{0} / 2 L_{c}$. When only one burner $(N=1)$ is considered, the dispersion relation (Eq. (4)) can be written explicitly,

$$
\begin{gathered}
\left(\Gamma_{1} \Gamma_{4}-\Gamma_{2} \Gamma_{3}\right) \sin \left(2 k L_{c}\right) \sin \left(2 k_{u} L_{p}\right) \\
+2 \Gamma_{1}\left[1-\cos \left(2 k L_{c}\right)\right] \sin \left(2 k_{u} L_{p}\right) \\
+2 \Gamma_{4}\left[1-\cos \left(2 k_{u} L_{p}\right)\right] \sin \left(2 k L_{c}\right) \\
+4\left[1-\cos \left(2 k L_{c}\right)\right]\left[1-\cos \left(2 k_{u} L_{p}\right)\right]=0,
\end{gathered}
$$

where $k=\omega / c^{0}$ and $k_{u}=\omega / c_{u}^{0}$ are the wave numbers, $c^{0}$ and $c_{u}^{0}$ are the sound speed in the burnt/unburnt gases (see Fig. 3 for notations). As illustrated in Fig. 7, the acoustic velocities $u^{\prime}$ in the plenum and in the chamber are coupled to the acoustic pressure through the burner and the flame. These interactions are modeled by four coupling parameters (Eq. (7)),

$$
\left\{\begin{array}{l}
\Gamma_{1}=-\frac{S_{i}}{2 S_{p}} \operatorname{cotan}\left(k_{u} L_{i}\right) \\
\Gamma_{2}=\frac{S_{i}}{2 S_{p}} \frac{1}{\sin \left(k_{u} L_{i}\right)} \\
\Gamma_{3}=\frac{S_{i} \rho^{0} c^{0}}{2 S_{c} \rho_{u}^{0} c_{u}^{0}} \frac{1+n^{j \omega \tau}}{\sin \left(k_{u} L_{i}\right)} \\
\Gamma_{4}=-\frac{S_{i} \rho^{0} c^{0}}{2 S_{c} \rho_{u}^{0} c_{u}^{0}}\left(1+n^{j \omega \tau}\right) \operatorname{cotan}\left(k_{u} L_{i}\right),
\end{array}\right.
$$

where $\Gamma_{1}$ is the coupling parameter associated with the plenum/burner junction, and $\Gamma_{4}$ with the chamber/burner junction. $\Gamma_{2}$ and $\Gamma_{3}$ control the interaction between the two annular cavities. Note that only the coupling parameters of the chamber $\left(\Gamma_{3}\right.$ and $\left.\Gamma_{4}\right)$ depend on the $n-\tau$ flame model. This situation corresponds to a pressure coupling: for example, if the azimuthal mode imposes a pressure node in the chamber (i.e., $p_{c}^{\prime}=0$ ), then the interactions $\Gamma_{2} p_{C}^{\prime}$ and $\Gamma_{4} p_{C}^{\prime}$ are null, independently of the coupling parameter values. This simple case allows the identification of three situations, for which the conclusion still holds for larger number of burners: 


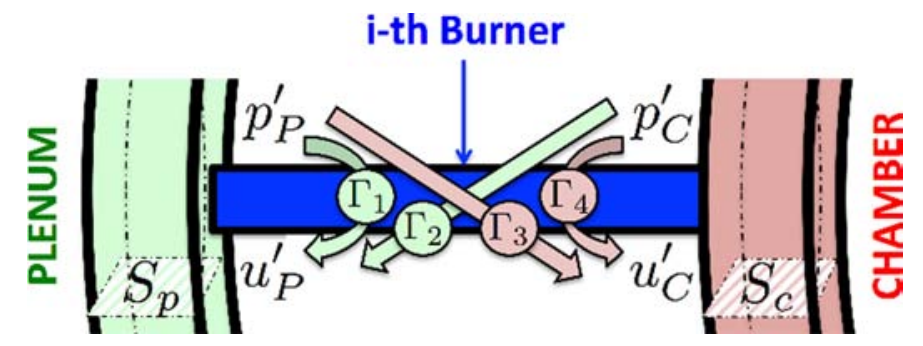

FIG. 7. Zoom on the burner connecting the annular plenum and the annular chamber. Acoustic pressure $\left(p^{\prime}\right)$ and velocity $\left(u^{\prime}\right)$ in the plenum (subscript $P$ ) and in the chamber (subscript $C$ ) are coupled. These interactions are modeled by four coupling parameters $\Gamma_{k=1 \ldots 4}$.

- Fully decoupled (FD) modes: When all coupling parameters $\Gamma_{i}$ are null, solutions of Eq. (6) of order $m$ are $f_{c}^{0}=m c^{0} / 2 L_{c}$ (pure azimuthal decoupled mode in the chamber) or $f_{p}^{0}=m c_{u}^{0} / 2 L_{p}$ (pure azimuthal decoupled mode in the plenum).

- Weakly coupled (WC) modes ((a) in Fig. 8): When coupling factors are not null but satisfy $\left\|\Gamma_{k=1 . .4, i}\right\| \ll 1$, solutions are close to the FD modes and can be searched as $f_{c}=f_{c}^{0}+\delta f$ and $f_{p}=f_{p}^{0}+\delta f$. A Taylor expansion of the dispersion relation (Eq. (4)) yields the solutions in the case where the two annular cavities are not naturally coupled, i.e., when $f_{p}^{0}$ and $f_{c}^{0}$ are not multiple of each other,

$$
f_{c}=\frac{m c^{0}}{2 L_{c}}-\frac{c^{0} \Gamma_{4}^{0}}{4 \pi L_{c}} \text { and } f_{p}=\frac{m c_{u}^{0}}{2 L_{p}}-\frac{c_{u}^{0} \Gamma_{1}^{0}}{4 \pi L_{p}} .
$$

The $f_{p}$ and $f_{c}$ modes correspond to "plenum" (WC plenum modes in Fig. 8, left) or "chamber" (WC chamber modes in Fig. 8, right) modes.

This result obtained for $N=1$ burner has been extended to configurations with $N$ burners as follows: ${ }^{71,72}$ for the WC chamber mode, the frequency scales like $f_{c}=\frac{m c^{0}}{2 L_{c}}-\frac{c^{0} N \Gamma_{4}^{0}}{4 \pi L_{c}} . \Gamma_{4}^{0}$ is the evaluation of the coupling parameter $\Gamma_{4}$ at the frequency of the corresponding FD mode,

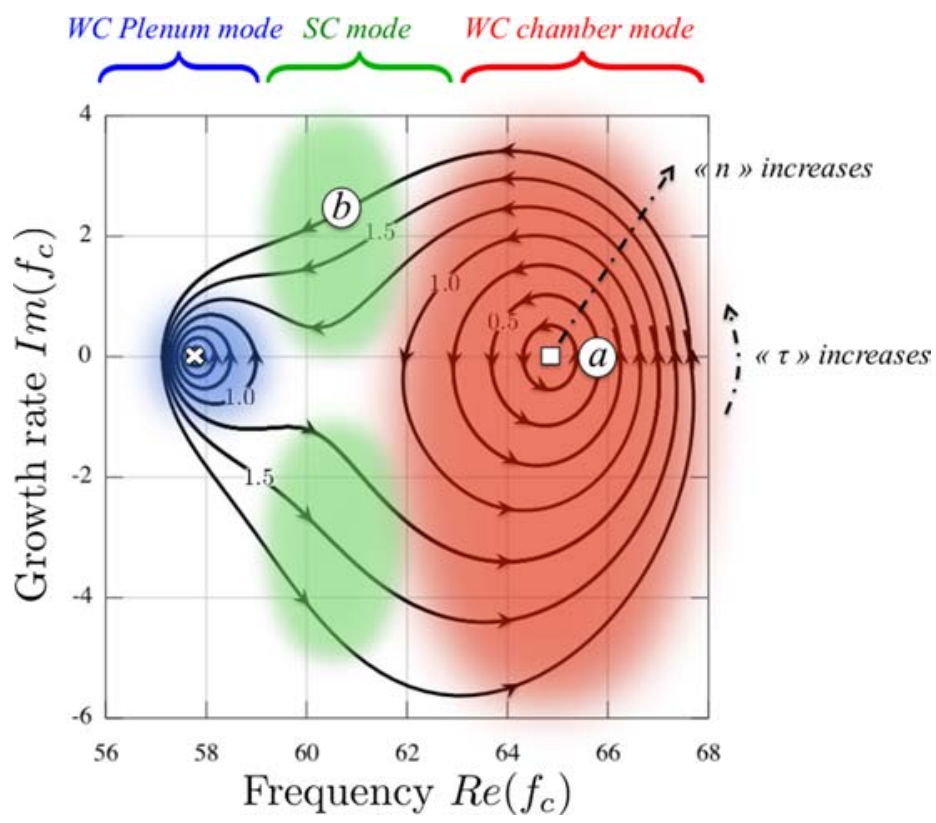

FIG. 8. Stability map $\left\{\operatorname{Re}\left(f_{c}\right), \operatorname{Im}\left(f_{c}\right)\right\}$ of a PBC configuration with $N=4$ burners where the FTF parameters are varied: $n$ from 0.25 to 1.75 and $\tau / \tau_{c}^{0}$ from 0 to 1.0. It highlights the bifurcation between WC and SC modes at $n=1.25$. FD modes are displayed by $\times$ (plenum) and $\square$ (chamber). 


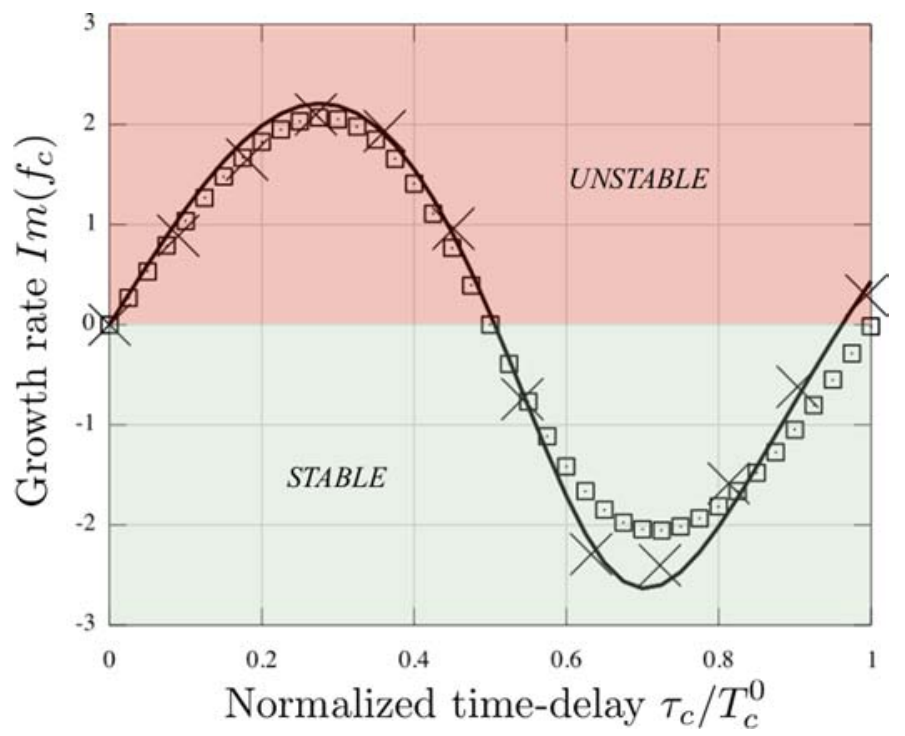

FIG. 9. Growth rate $\operatorname{Im}\left(f_{c}\right)$ of the first chamber mode $(m=1)$ in a PBC combustor with $N=4$ burners where $n=1.57$ while the time-delay is varied from 0 to $\tau_{c}^{0}$, where $\tau_{c}^{0}=1 / f_{c}^{0}$. The growth rate is estimated by the full analytical formula $f_{c}=\frac{c^{0}}{2 L_{c}}+\frac{c^{0} N \Gamma_{4}^{0}}{4 \pi L_{c}}(\square)$, a semi-analytical resolution (-) of the dispersion relation (Eq. (4)), and a $3 D$ Helmholtz solver $(\times)$.

i.e., $f=f_{c}^{0}$. The flame delay $\tau$ is normalized by the period of the first azimuthal mode $\tau_{p}^{0}$ or $\tau_{c}^{0}$. This analytical expression is validated against a semi-analytical method as well as a $3 \mathrm{D}$ Helmholtz solution in Fig. 9.

- Strongly coupled (SC) modes ((b) in Fig. 8): When the weakly coupled assumption $\left\|\Gamma_{k=1 . .4, i}\right\|$ $\ll 1$ is not satisfied, the two annular cavities can couple and oscillate at a frequency which is not close to $f_{p}^{0}$ or $f_{c}^{0}$. In this case, the acoustic mode cannot be identified as "plenum" or "chamber" mode since the whole combustor resonates: a bifurcation occurs (SC modes in Fig. 8, middle). Only partially analytical solutions can be obtained in such a situation. In particular, SC modes in Fig. 8 are obtained by solving the dispersion relation (Eq. (4)) numerically.

This analytical study ${ }^{30}$ allows the classification of azimuthal modes as "fully decoupled," "weakly coupled," or "strongly coupled" modes. For the two first categories, the acoustic mode is present in only one annular cavity, and therefore can be identified as "plenum" or "chamber" modes (Fig. 10, top). However, when the flames tune one annular cavity so that it matches the resonant frequency of the second cavity, a bifurcation in the stability map occurs: for this case, the mode cannot be classified as "plenum" or "chamber" modes since the whole combustor resonates (Fig. 10, bottom). Note that each trajectory (— in Fig. 8) of the stability map requires 200 simulations, which leads to more than 1000 simulations, performed here using the ATACAMAC network tool developed by Bauerheim et al., ${ }^{30}$ to investigate this plenum/chamber coupling. Therefore, this type of study is possible only because of the development of recent theoretical tools, ${ }^{30,61,66,70}$ since performing thousands $3 \mathrm{D}$ Helmholtz simulations of complete $360^{\circ}$ combustors still constitutes an important computational effort today. Note however that applying a Floquet-Bloch theory allows a drastic cost reduction of $360^{\circ}$ Helmholtz simulations by computing only one sector of a symmetric annular combustor. Unfortunately, only approximations of the solutions can be achieved when applying this technique to weakly asymmetric cases. ${ }^{80}$

A phase-lag $\beta$ between the annular chamber and the plenum is observed in Fig. 10 for both weakly and strongly coupled regimes: this phase-lag is controlled by the time-delay $\tau$. In the WC case, $\tau / \tau_{c}^{0}=0$ leads to a phase-lag $\beta=\pi$, whereas in the SC case, $\tau / \tau_{c}^{0}=0.5$ yields a phase-lag $\beta=\pi / 2$. Bourgouin et al. ${ }^{66}$ have investigated this phenomenon analytically on the MICCA annular experiment. They found that for a WC plenum mode, $\tau / \tau_{c}^{0}=0$ leads to $\beta=0$ 


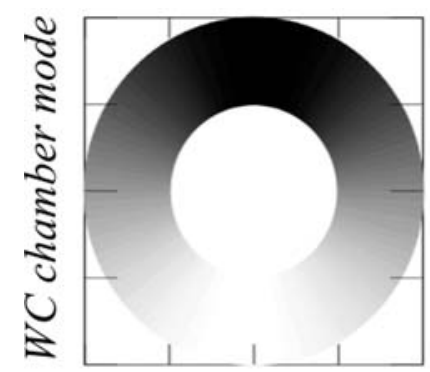

CHAMBER

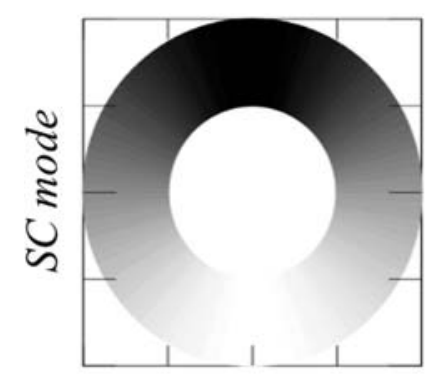

CHAMBER

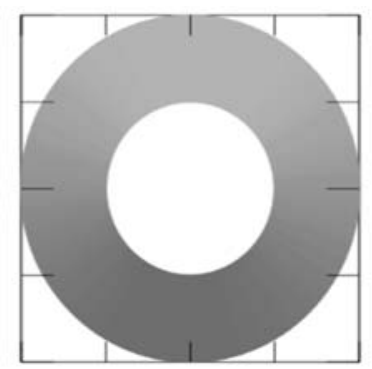

PLENUM

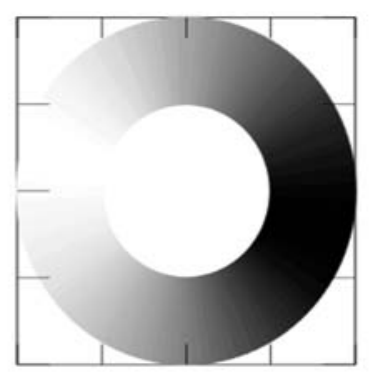

PLENUM

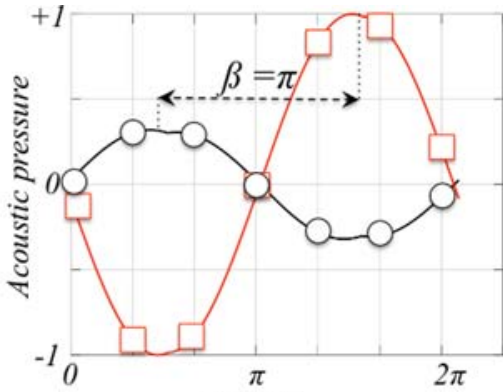

Angle $\theta$

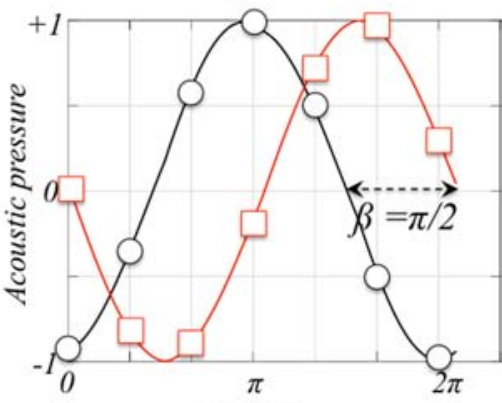

Angle $\theta$

FIG. 10. Mode structure for $N=4$ burners in the chamber (left and $\square$ ) and in the plenum (middle and $\circ$ ) for a WC chamber mode (top row- $n=0.5 ; \tau / \tau_{c}^{0}=0$ ) and SC mode (bottom row- $n=1.75 ; \tau / \tau_{c}^{0}=0.5$ ).

(or $\pi$ if $\operatorname{Re}\left(f_{c}\right)>\operatorname{Re}\left(f_{c}^{0}\right)$ as in Fig. 10) and then decreases with the time-delay. For SC modes, network models show that the maximum coupling is obtained when $\beta=\pi / 2$ for this $N=4$ burner configuration. ${ }^{30}$

\section{B. Degenerate vs. non-degenerate modes}

A specificity of azimuthal modes is their apparent natural degeneracy: an acoustic mode in a simple annular chamber can turn clockwise or counter-clockwise, leading to two possible modes for each eigenfrequency. The two components of the azimuthal mode are usually "degenerate," 37 meaning that they have the same frequencies and growth rates. Understanding when and how azimuthal modes become non-degenerate, ${ }^{37,72}$ i.e., with different frequencies or growth rates, brings up many other issues such as symmetry breaking which are discussed below.

\section{Mixing different burner types}

Frequencies and stability maps obtained in Section III A have been validated against $3 D$ acoustic simulations in many applications, from academic combustors ${ }^{30,71,72}$ to industrial gas turbines. ${ }^{70,81}$ However, only axisymmetric combustors were investigated. Recently, the need of considering non-symmetric combustors has appeared, mainly to investigate novel passive techniques to control azimuthal modes. For instance, introducing Helmholtz resonators ${ }^{69,79}$ baffles ${ }^{82}$ or different types of burners ${ }^{67,83}$ may damp unstable modes but also break the rotational symmetry of the configuration. For the latter option, choosing which burner types must be mixed in a chamber and how to distribute them along the azimuthal direction to damp azimuthal modes are an open topic that analytical studies can help clarify.

Network models applied to a burner-chamber (BC) configuration ((b) in Fig. 6) can be used to extend the results of Section III A to non-symmetric combustors containing an arbitrary number of burners $N .^{72}$ Burners can differ from each other because of their geometrical characteristics, which may lead to different FTFs for each flame. This yields different coupling parameters $\Gamma_{4, i}$ (in a BC configuration, only one type of coupling parameter exists, so that the subscript 4 can be omitted). 
For weakly coupled modes, the frequencies of the azimuthal mode of order $m$ are

$$
f_{m}^{ \pm}=\frac{m c^{0}}{2 L_{c}}-\frac{c^{0}}{4 \pi L_{c}}\left(\Sigma_{0} \pm \mathcal{S}_{0}\right)
$$

where

$$
\left\{\begin{array}{l}
\Sigma_{0}=\sum_{i=1}^{N} \Gamma_{i}^{0} \\
\mathcal{S}_{0}=\sqrt{\sum_{i, j=1}^{N} \Gamma_{i}^{0} \Gamma_{j}^{0} \cos \left(\frac{4 m \pi}{N}(j-i)\right)} .
\end{array} .\right.
$$

This result demonstrates that the stability of non-symmetric annular combustors is controlled by two parameters:

- the "coupling strength" $\Sigma_{0}$, which is the sum of all coupling parameters of the system, and is independent of the pattern used to distribute the burners in the chamber. It corresponds to a symmetric effect associated to a "mean flame" FTF. For instance, in a case with $N=24$ burners with two types of burners, characterized by a coupling parameter $\Gamma_{A}^{0}$ (4 flames) and $\Gamma_{B}^{0}$ (20 flames), the coupling parameter is $\Sigma_{0}=4 \Gamma_{A}^{0}+20 \Gamma_{B}^{0}$. Thus, it is equivalent to use 24 identical burners with a coupling parameter $\Gamma^{0}=\frac{4}{24} \Gamma_{A}^{0}+\frac{20}{24} \Gamma_{B}^{0}$. Consequently, this parameter can be changed, for example, by using 8 burners of type $A$ and 16 of type $B$, to stabilize one or multiple azimuthal modes (Fig. 11). ${ }^{72}$

- the "splitting strength" $\mathcal{S}_{0}$ is the quantity which "splits" the two azimuthal mode frequencies $f_{m}^{+}$and $f_{m}^{-}$(sign \pm in Eq. (10)). A convenient form of this parameter is obtained by using the spatial Fourier transform of the coupling parameter distribution $\gamma$ :

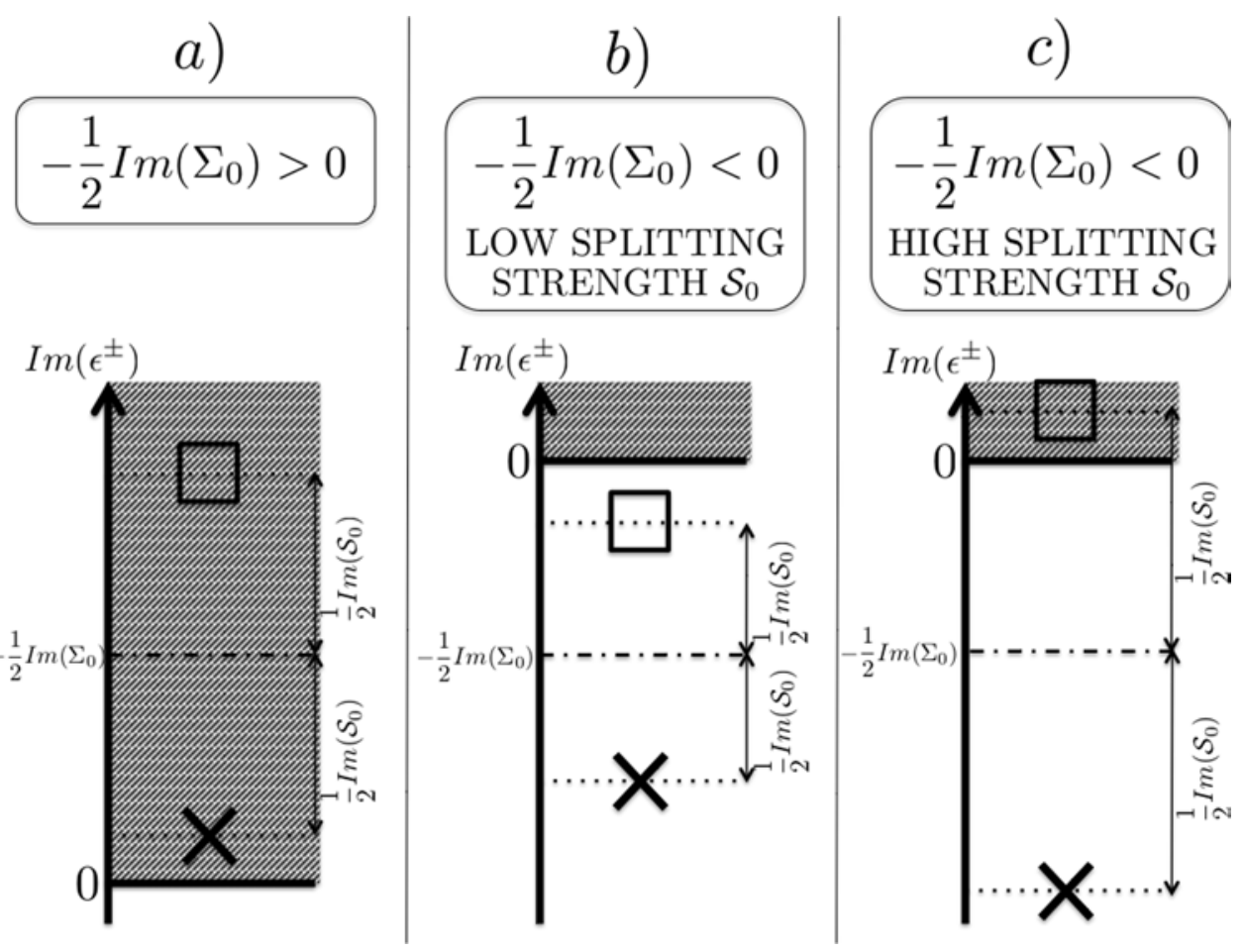

FIG. 11. Three stability cases given by the normalized growth rate $\operatorname{Im}\left(\epsilon^{ \pm}\right)=\frac{2 \pi L_{c}}{c^{0}} \operatorname{Im}\left(f^{ \pm}\right)$of the two components $(\times$and $\square)$ depending on the coupling strength $\Sigma_{0}$ and splitting strength $\mathcal{S}_{0}$. (a) The coupling strength leads to an unstable mode. (b) The coupling strength leads to a stable mode since the splitting strength is low. (c) The coupling strength should lead to a stable mode, but because of a large splitting strength, the mode is unstable (one of its components is unstable). 


$$
\mathcal{S}_{0}=\sqrt{\gamma(2 m) \gamma(-2 m)}, \text { where } \gamma(k)=\sum_{i=1}^{N} \Gamma_{i}^{0} e^{-\frac{j 2 k \pi i}{N}} .
$$

It shows that only specific patterns can affect the azimuthal mode stability. They correspond to the $\pm 2 m$ th Fourier coefficient $\gamma$ of the coupling parameter or heat-release distribution. Therefore, unlike the coupling strength $\Sigma_{0}$, this parameter can be changed by modifying the pattern of the burner types along the annular chamber.

Note that since the splitting strength damps one mode but always increases the growth rate of the second one by $\operatorname{Im}\left(\mathcal{S}_{0}\right) / 2$, using a splitting strength with a non-null imaginary part always makes the system less stable (Fig. 11): ${ }^{72,84}$ according to theory, combining different burners is not a solution to control one mode. However, mixing different burner types can become useful to modify $\Sigma_{0}$ in order to control multiple modes, or one acoustic mode at different operating points. Nevertheless, using different burner types leads to an undesired side effect: a splitting effect which makes the mode less stable (Fig. 11). This drawback can be avoided by rearranging the burners along the annular chamber to reduce the splitting strength. For instance, when only two burner types are used, the splitting strength reduces to

$$
\left\|\mathcal{S}_{0}\right\|=\overbrace{2 \mathcal{K}}^{\text {Imposed by the pattern }} \underbrace{\left\|\Gamma_{A}^{0}-\Gamma_{B}^{0}\right\|,}_{\text {Imposed by the difference between burner types }}
$$

where $\mathcal{K}$ is a constant which only depends on the pattern. ${ }^{72}$

Equation (12) shows that the splitting strength increases when the difference between the two burner types $\left\|\Gamma_{A}^{0}-\Gamma_{B}^{0}\right\|$ increases, which is necessary to control modes by changing significantly $\Sigma_{0}$. But it also reveals that the splitting strength can be reduced by changing the pattern, i.e., $\mathcal{K}$ : an optimization procedure over all patterns possible can be performed to find the pattern which minimizes $\mathcal{K}$, in order to suppress this side-effect due to splitting. Determining all patterns which lead to $\mathcal{K}=0$ is a complicated mathematical exercise in general. For example, in a 24 burner machine, where 4 "different" (Type 2, white in Fig. 12) burners are inserted in the annulus ${ }^{67,83}$ as two pairs of burners separated by an angle $\Delta \theta$, analytical results show that specific rearrangements of burners can lead to $\mathcal{K}=0$, and therefore a zero splitting effect (Fig. 12, when $\Delta \theta=75^{\circ}$ or $\Delta \theta=225^{\circ}$ for the first azimuthal mode $\left.m=1\right) .^{72}$ Again, such an optimization procedure is made possible only because of recent progress in theoretical tools for thermoacoustics, since computing all patterns with a $3 D$ Helmholtz solver is not feasible yet (more than 2000 patterns exist for a 24 burner configuration with $N_{A}=4$ burners of type $A$, and more than 77000 for $N_{A}=6$ ).

\section{Mode degeneracy and symmetry breaking}

Annular combustors are not the only example in physics where symmetry breaking plays a role: similar problems are found for azimuthal modes in a wide range of physical problems where

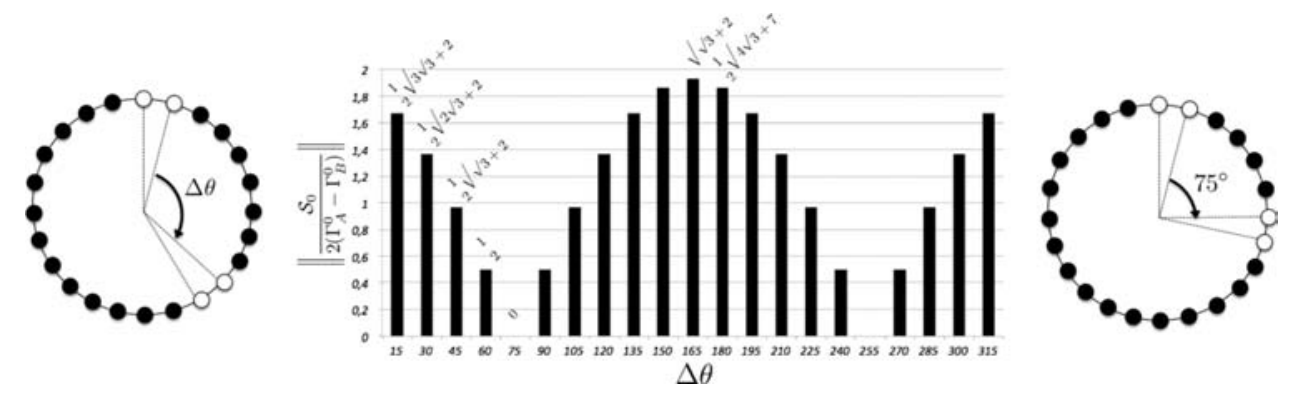

FIG. 12. The reduced splitting strength $\mathcal{K}$ for several patterns where two burners of type $A$ are kept together at the same place, and two others are changed azimuthally so that only one parameter controls the burner distribution: the angle $\Delta \theta$. Optimal patterns are found analytically to reduce the splitting strength of the first azimuthal mode $m=1$ : for example, using $\Delta \theta=75^{\circ}$ yields $\mathcal{K}=0$ (right). 
rotational symmetry is broken (seismic waves in a rotating giant star, ${ }^{85}$ oscillations of a rotating or aspheric liquid droplet, ${ }^{86,87}$ vibration of non-symmetric molecules, ${ }^{88}$ etc.). For instance, Davey and Salwen ${ }^{89}$ investigated the linear stability of hydrodynamic circumferential modes in both circular and elliptic pipes. They show analytically that the circular problem has double eigenvalues $f_{0}$ (called a "degenerate pair"), while the ellipticity of the latter configuration splits the doublets into two distinct eigenvalues $f_{0} \pm \Delta f / 2$ (called "non-degenerate" eigenvalues). They mentioned that the imaginary part of the splitting frequency $\Delta f$ being non-zero, the ellipticity always makes the flow less stable. ${ }^{89}$

For annular combustors, splitting can be introduced on purpose or be a consequence of undesired differences in burners due to manufacturing tolerances. When splitting is introduced on purpose by the engine designers, the objective is usually to damp unstable modes: symmetry breaking is created by mixing different burner types (Section III B 1): this type of symmetry breaking is called "Geometrical Symmetry" (GS) breaking. ${ }^{71}$ Other types of symmetry breaking can be investigated to highlight their effect on azimuthal mode stability. For instance, the introduction of a mean swirl motion by swirlers or effusive plates, called "Flow Symmetry" (FS) breaking, has been investigated recently. ${ }^{71}$ It shows that an additional splitting effect due to the mean flow is present, and can interact non-linearly with the splitting due to the GS breaking $\mathcal{S}_{0}$,

$$
\mathcal{S}_{M}=\sqrt{\mathcal{S}_{0}^{2}+4 \pi^{2} M_{\theta}^{2}},
$$

where $\mathcal{S}_{M}$ is the total splitting strength and $M_{\theta}$ is the mean azimuthal Mach number. The analytical solution of the dispersion equation allows to analyse three typical situations:

- GS breaking: When $\left\|\mathcal{S}_{0}\right\| \gg 2 \pi M_{\theta}$, the splitting is mainly affected by the difference between burners, i.e., by GS breaking. When $\operatorname{Im}\left(\mathcal{S}_{0}\right) \neq 0$, splitting always makes the mode less stable, as mentioned by Davey and Salwen ${ }^{89}$ for hydrodynamic modes. Note that this conclusion may differ for strongly coupled modes. ${ }^{90}$

- FS breaking: If $\left\|\mathcal{S}_{0}\right\| \ll 2 \pi M_{\theta}$, the symmetry breaking is driven by the flow itself. However, since $M_{\theta}$ is real, the resulting splitting strength has a null imaginary part: the mode is non-degenerate but the stability is unchanged.

- FS+GS breaking: When both $\mathcal{S}_{0}$ and $M_{\theta}$ are significant, the two splitting effects (FS and GS) interact non-linearly (the resulting splitting strength is not just the sum of $\mathcal{S}_{0}$ and $2 \pi M_{\theta}$ ): FS breaking can increase or decrease the splitting effect and affect the stability of the configuration.

In addition with this analysis, Bauerheim, Cazalens, and Poinsot ${ }^{71}$ show that the mean azimuthal flow affects not only the splitting strength $\mathcal{S}_{0}$, but also the coupling strength $\Sigma_{0}$ and thus the stability. Indeed, the mean flow changes the propagation and interaction characteristics of the annular combustor, which (1) modifies the phase-lag in the flame response $\phi=\omega_{0} \tau$, since $\omega_{0}$ varies like $(1 \pm M)$ and (2) enhances the coupling parameters, and therefore the coupling strength, by a factor proportional to $3 M^{2}$. Moreover, when the flame response to acoustics depends on the frequency, the time-delay $\tau=\tau(\omega)$ is affected by the mean flow.

This analytical study suggests that symmetry breaking is an essential element of all control strategies for azimuthal modes (using, for example, baffles or Helmholtz resonators to damp acoustic modes). Note however that other engine modifications can also produce symmetry breaking: introducing additional effusive plates will induce a mean swirl and a FS breaking effect; adding spark plugs or ignition injectors will introduce GS breaking. Even if thermoacoustics was not the reason for these modifications, they will be affected by these changes. Moreover, symmetry breaking effects can also be obtained in systems which are supposed to be perfectly axisymmetric, since manufacturing tolerances can lead to slightly different burners. This topic is investigated in Section III E. 


\section{Linear vs. non-linear flame response}

Section III B has shown the role of symmetry breaking for azimuthal modes in annular combustors. However, this analysis remains in the linear regime. This section analyzes recent developments and results regarding symmetry breaking in the non-linear regime. In particular, investigating the mode structure of circumferential acoustic modes with modal coupling and non-linear flame response ${ }^{61,62}$ can provide information on the limit cycle, which can be compared with experimental observations. $^{38,43,65,66,82}$

\section{Perfectly axisymmetric case}

Noiray et al. propose a model to analyze non-linear effects on azimuthal modes: in this study, a simple axisymmetric annulus ((a) in Fig. 6) is considered (so that $\Psi_{m}=\cos (m \theta)$ and $\left.\Psi_{-m}=\sin (m \theta)\right)$ with a distributed heat release along the azimuthal direction $\dot{\omega}_{T}{ }^{\prime}(t, \theta)$. First, when the acoustic damping $\alpha$ and a simple linear flame response model $\dot{\omega}_{T}{ }^{\prime}(t, \theta)=\frac{n}{\gamma-1} p^{\prime}(t, \theta)$ are introduced, the truncated Galerkin method yields decoupled equations for each mode of order $m$,

$$
\frac{d^{2} \eta_{m}}{d t^{2}}+\alpha \frac{d \eta_{m}}{d t}+\omega_{m}^{2} \eta_{m}=n \frac{d \eta_{m}}{d t}
$$

Equation (14) corresponds to a classical harmonic resonator of frequency $\omega_{m}$ and growth rate $(n-\alpha) / 2$. Thus, similarly to the previous linear study, no coupling between the two components $\pm m$ of the azimuthal mode is observed: the mode nature is undetermined.

Now, when a non-linear flame response $\dot{\omega}_{T}^{\prime}(t)=\frac{n}{\gamma-1} p^{\prime}(t)-\frac{\kappa}{\gamma-1} p^{\prime}(t)^{3}$ is introduced, the equation becomes

$$
\begin{gathered}
\frac{d^{2} \eta_{m}}{d t^{2}}+\alpha \frac{d \eta_{m}}{d t}+\omega_{m}^{2} \eta_{m}=n \frac{d \eta_{m}}{d t} \\
-\frac{3}{4} \kappa\left(\frac{d \eta_{m}}{d t}\left[3 \eta_{m}^{2}+\eta_{-m}^{2}\right]+2 \frac{d \eta_{-m}}{d t} \eta_{m} \eta_{-m}\right),
\end{gathered}
$$

with a similar equation for $\eta_{-m}$, the two equations corresponding actually to a model for two coupled Van der Pol oscillators. ${ }^{61}$ For this approximate analysis, Noiray, Bothien, and Schuermans ${ }^{61}$ assume that modal amplitudes can be written as $\eta_{m}=\mathcal{A}(t) \cos \left(\omega_{m} t\right)$ and $\eta_{-m}=\mathcal{B}(t) \sin \left(\omega_{m} t\right)$, where $\mathcal{A}(t)$ and $\mathcal{B}(t)$ are varying slowly in time compared to the acoustic oscillations. It leads to

$$
\left\{\begin{array}{l}
\frac{d \mathcal{A}}{d t}=\frac{1}{2}(n-\alpha) \mathcal{A}-\frac{\kappa}{32}\left(9 \mathcal{A}^{2}+3 \mathcal{B}^{2}\right) \mathcal{A} \\
\frac{d \mathcal{B}}{d t}=\frac{1}{2}(n-\alpha) \mathcal{B}-\frac{\kappa}{32}\left(9 \mathcal{B}^{2}+3 \mathcal{A}^{2}\right) \mathcal{B}
\end{array} .\right.
$$

In contrast with linear stability analysis, the two components of the azimuthal modes interact due to the third order non-linear flame model. Limit cycles are searched as fixed points of the system (16), i.e., $\frac{d \mathcal{A}}{d t}=\frac{d \mathcal{B}}{d t}=0$. Nine fixed points $\left(\mathcal{A}_{0}, \mathcal{B}_{0}\right)$ of Eq. (16) exist; ${ }^{61}$ however, they are not necessarily stable. A perturbation analysis (linearization around the fixed points, e.g., $\mathcal{A}$ $\left.=\mathcal{A}_{0}+\delta \mathcal{A}\right)$ leads to the dynamic behavior of the acoustic modes for the several limit cycles found,

$$
\frac{d}{d t}\left(\begin{array}{c}
\delta \mathcal{A} \\
\delta \mathcal{B}
\end{array}\right)=M_{d y n}\left(\mathcal{A}_{0}, \mathcal{B}_{0}\right)\left(\begin{array}{c}
\delta \mathcal{A} \\
\delta \mathcal{B}
\end{array}\right) .
$$

The sign of the two eigenvalues of $M_{d y n}$ depends on the mode structure of the limit cycle (values of $\mathcal{A}_{0}$ and $\mathcal{B}_{0}$ ). It provides the dynamic behavior of the corresponding limit cycle:

- Linear phase $\left(\mathcal{A}_{0}=\mathcal{B}_{0}=0\right)$ : the two associated eigenvalues of the linearized dynamic system are $\lambda=\frac{1}{2}(n-\alpha)$, so that considering $n>\alpha$ this solution is a "repeller": the mode is unstable and grows with time to deviate from this non-oscillating state: it corresponds to the linear growth of the azimuthal mode.

- Standing modes $\left(\left(\mathcal{A}_{0}=0\right.\right.$ and $\left.\mathcal{B}_{0} \neq 0\right)$ or vice-versa $)$ : The two eigenvalues have opposite signs, thus these solutions are saddle points. 
- Spinning modes $\left(\mathcal{A}_{0}= \pm \mathcal{B}_{0} \neq 0\right)$ : The eigenvalues of the linearized dynamical system are proportional to $\alpha-n$, so that for a linearly unstable mode this solution is an "attractor."

Consequently, ${ }^{35,61}$ show that a linearly unstable azimuthal mode in a uniform annular system will always result in a spinning wave $\left(\mathcal{A}_{0}= \pm \mathcal{B}_{0}\right)$ for the non-linear flame model considered in this work.

\section{Symmetry breaking effect on azimuthal mode dynamics}

Noiray, Bothien, and Schuermans ${ }^{61}$ have then extended this non-linear analysis to cases where the heat release distribution is not uniform along the annulus in order to break its symmetry: in the previous flame model, the constant $\beta$ is replaced by an azimuthal Fourier decomposition $n=n_{0}\left(1+\sum_{m=1}^{M} C_{m} \cos (m \theta)\right)$, which yields the linear component of the heat release source term $n_{0} \frac{d \eta_{ \pm m}}{d t}\left(1 \pm \frac{C_{2 m}}{2}\right)$. Because of this symmetry breaking, the dynamical system is changed and becomes

$$
\left\{\begin{array}{l}
\frac{d \mathcal{A}}{d t}=\frac{1}{2}\left(n_{0}\left[1+\frac{C_{2 m}}{2}\right]-\alpha\right) \mathcal{A}-\frac{\kappa}{32}\left(9 \mathcal{A}^{2}+3 \mathcal{B}^{2}\right) \mathcal{A} \\
\frac{d \mathcal{B}}{d t}=\frac{1}{2}\left(n_{0}\left[1-\frac{C_{2 m}}{2}\right]-\alpha\right) \mathcal{B}-\frac{\kappa}{32}\left(9 \mathcal{B}^{2}+3 \mathcal{A}^{2}\right) \mathcal{B}
\end{array} .\right.
$$

Again, this system has nine fixed points $\left(\mathcal{A}_{0}, \mathcal{B}_{0}\right)$ which depend now on the coefficient $C_{2 m}$. A perturbation analysis similar to Eq. (17) leads to eigenvalues of the dynamic system, which describes the stability of the limit cycle considered. First, for the linear phase (case corresponding to the fixed point $\mathcal{A}_{0}=\mathcal{B}_{0}=0$ ), it is found that the mode is unstable if

$$
n_{0}\left[1 \pm \frac{C_{2 m}}{2}\right]-\alpha>0 \text {. }
$$

This condition is similar to the condition obtained by the linear acoustic network (Section III B) where $n_{0}$ is viewed as a "coupling strength" associated with a mean flame effect (symmetric effect), and $\pm n_{0} C_{2 m} / 2$ is similar to a "splitting strength." Note however that in the linear analysis of Section III B, the splitting coefficient $\mathcal{S}_{0}$ is more complex since it involves both the $C_{2 m}$ and $C_{-2 m}$ coefficients describing the heat release distribution, but also the burners geometry, as well as the mean swirl effect $2 \pi M_{\theta}$.

The two standing modes $\left(\mathcal{A}_{0}=0\right.$ or $\left.\mathcal{B}_{0}=0\right)$ are still fixed points of the dynamical system with eigenvalues $\frac{1}{3}\left(n_{0}\left[1 \pm C_{2 m}\right]-\alpha\right)$ and $\alpha-n_{0}\left[1 \mp \frac{C_{2 m}}{2}\right]$. Therefore, for low "splitting strength," standing modes are still a "repeller," but for large "splitting strength," they become an "attractor."

When symmetry is broken, spinning modes are not a fixed point of the dynamical system anymore $\left(\mathcal{A}_{0} \neq \mathcal{B}_{0}{ }^{61}\right)$ : they correspond to mixed modes, which are "attractors" for low "splitting strength" and become "saddle points" for large symmetry breaking.

In other words, this non-linear analysis proves that only the component of order $\pm 2 m$ of the heat-release distribution contributes to the stability and dynamics of azimuthal modes. When the configuration is perfectly symmetric, spinning modes appear. However, when GS breaking occurs, characterized by a large "splitting strength" $C_{2 m}$, standing modes should be observed during the limit cycles. However, these results do not necessary imply that these behaviors are stationary even when they involve "attractors," as observed in academic experiments, ${ }^{38,39}$ industrial gas turbines, ${ }^{34,64}$ or simulations of complete $360^{\circ}$ combustors ${ }^{12,14,46,77}$ (see Section III D).

\section{Symmetry breaking induced by non-linear flame response}

Sections III B and III C demonstrate that the splitting strength is the quantity which controls most of the azimuthal modes nature in annular chambers. The next question is to evaluate which mechanism can create significant values of this parameter. Fuel staging and mixing burner types are typical mechanisms leading to symmetry breaking. ${ }^{61,67,70-72,83}$ But it has also been shown recently that non-linearities themselves (i.e., non-linear flame response to acoustics) can break the rotational 
symmetry of the combustor by producing a non-uniform heat release distribution: in this situation, symmetry breaking (and large values of the splitting strength) are created by the flames themselves.

Ghirardo, Juniper, and Moeck ${ }^{63}$ have extended the previous non-linear analysis to arbitrary flame describing functions $Q(\mathcal{A}, \omega)=G(\mathcal{A}, \omega) e^{j \Phi(\mathcal{A}, \omega)}$ in a perfectly axisymmetric combustor (i.e., a simple $1 D$ annulus where the flame response is described by a gain $G$ and a phase-lag $\Phi$ depending on both the angular frequency $\omega$ and the amplitude of the oscillations $\mathcal{A}$ ). Similar to Noiray, Bothien, and Schuermans, ${ }^{61}$ they found that spinning and standing modes lead to different criteria regarding the stability of limit cycles:

- Spinning modes: When considering slow variations of the spinning wave amplitude $\mathcal{A}(\theta, t)$ compared with the acoustic oscillations $\left(\mathcal{A}(\theta, t)=\mathcal{A}^{s p}\right.$ is uniform for a spinning mode, (a) in Fig. 13), the stability of the associated limit cycle is governed by only one criterion,

$$
\operatorname{Re}\left[\frac{d Q\left(\mathcal{A}^{s p}, \omega\right)}{d \mathcal{A}^{s p}}\right]<0 .
$$

Ghirardo, Juniper, and Moeck ${ }^{63}$ proved that this condition can be also obtained by differentiating the classical Rayleigh criterion. It allows the extraction of the physical explanation of the limit cycle stability: since the configuration is perfectly symmetric and all flames have a similar response to acoustics ((a) in Fig. 13), the stability is obtained from an energy point of view, i.e., the limit cycle is stable if and only if the flame response decreases when the amplitude of the oscillation increases.

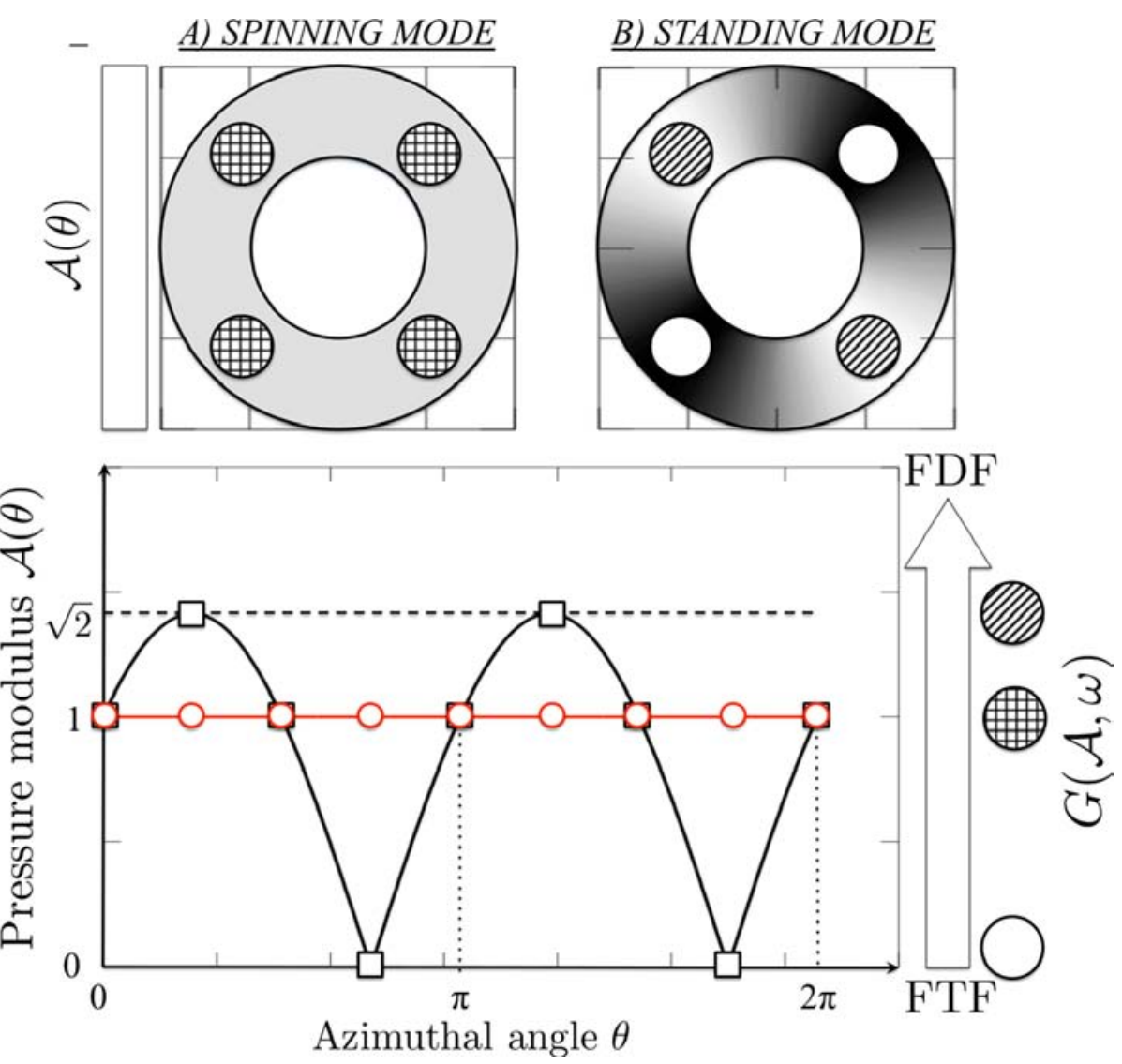

FIG. 13. Sketch of the pressure amplitude $\mathcal{A}(\theta)$ corresponding to $m=1$ for two different mode natures on a combustor with $N=4$ burners: (a) spinning modes $\mathcal{A}(\theta)=\mathcal{A}^{s p}$, in black and (b) standing modes $\mathcal{A}(\theta)=\mathcal{A}^{s t} \sqrt{1+\sin (2 \theta)}$, in red In the non-linear regime, the gain $G$ depends on the amplitude $\mathcal{A}(\theta)$ so that standing modes lead to symmetry breaking: FTF (empty circle) at pressure nodes and FDF (hatched circle) at pressure anti-nodes. 
- Standing modes: Compared with spinning modes, standing modes lead to a non-symmetric flame response in the non-linear regime ((b) in Fig. 13) because of its non-uniform pressure amplitude $\mathcal{A}(\theta, t)=\mathcal{A}^{s t} \sqrt{1+\sin (2 \theta)}$. Thus, considering flame models where the heat-release fluctuation is a non-linear function of pressure, flames located at pressure nodes are modeled via a linear FTF $(G(0, \omega)$ and $\Phi(0, \omega))$, whereas flames at pressure anti-nodes yield a nonlinear response (FDF). Consequently, a non-uniform distribution of heat-release is generated, as the one imposed a priori in the Noiray's analysis. Ghirardo, Juniper, and Moeck ${ }^{63}$ found that for standing modes, a condition based on the Rayleigh criterion (Eq. (20)) is necessary, but not sufficient for the stability of the limit cycle. A second condition appears,

$$
\int_{0}^{2 \pi} \operatorname{Re}\left[Q\left(\mathcal{A}^{s t} \sqrt{1+\sin (2 \theta)}, \omega\right)\right] \sin (2 m \theta) d \theta>0 .
$$

This condition involves the $2 m$ th spatial Fourier coefficient of the heat release distribution $\int_{0}^{2 \pi} \operatorname{Re}[Q] \sin (2 m \theta) d \theta$, as in the analytical expression of the "splitting strength" $\mathcal{S}_{0}$ (Eq. (11)): ${ }^{71,72}$ it can be explained by the rotational symmetry breaking induced by the non-uniform heat-release distribution of the standing modes ((b) in Fig. 13). This result can be extended to any azimuthal mode with a slowly moving nodal line, as observed for standing modes rotating slowly due to a mean swirling motion (i.e., where the characteristic time of the nodal line dynamics $\tau_{n l}$ is larger than the acoustic period $\tau_{c}^{0}: \tau_{n d} \ll \tau_{c}^{0}$ ). Moreover, a third stability condition also appears, ${ }^{63}$ which controls the nodal line orientation of a standing mode. Note that this condition vanishes when considering a configuration with a "continuous" rotational symmetry (compared with the "discrete" rotational symmetry for cases containing a finite number of burners), i.e., for an infinite number of burners with a distributed heat release. ${ }^{61}$ The last two conditions highlight the key role of symmetries for acoustic modes in annular combustors.

To support this conclusion, this splitting effect depending on the mode structure can be also obtained using network models by introducing $\mathrm{FDFs}^{46,76}$ which depend on the pressure $\left\|p^{\prime}(\theta)\right\|$ or longitudinal velocity $\left\|w^{\prime}(\theta)\right\|$ modulus (however no modal coupling can be incorporated so that the system's dynamic cannot be investigated). Note that for an azimuthal mode of order $m$, typically $p^{\prime}(\theta)=\sin (m \theta)$, one can prove that its modulus $\left\|p^{\prime}(\theta)\right\|$ contains a strong $\pm 2 m$ th Fourier coefficient, associated to the fact that the spatial period of $\left\|p^{\prime}\right\|$ is two times smaller than the period of $p^{\prime}$ (Appendix C in Ref. 46),

$$
\|\sin (m \theta)\|=\frac{2}{\pi} \sum_{k \in \mathbb{Z}} \frac{\sin ^{2}(k m \theta)}{k^{2}-1 / 4}=-\frac{2}{\pi} \sum_{k \in \mathbb{Z}} \frac{1}{4 k^{2}-1} e^{2 j k m \theta},
$$

where the first harmonic $k=1$ leads to spatial variations like $2 m \theta$ with an amplitude $2 / 3 \pi$, to be compared with $m \theta$ of the pressure oscillations. In other words, the non-linear flame response generated by a standing mode is non-uniform with a strong $2 m$ th component, which breaks the symmetry of the configuration and affects both stability and dynamics of the circumferential acoustic modes.

\section{Deterministic vs. probabilistic analysis}

Previous analytical studies (Sections III A-III C) provide significant results to understand stability, nature, and dynamics of azimuthal acoustic modes in annular combustors. However, they usually assume that the dynamics is governed by the acoustic mode itself. This is usually a good approximation in the linear regime, but it does not hold for non-linear regimes in highly turbulent annular combustors where turbulence may affect the flame and acoustics interactions. For instance, a detailed statistical analysis of the acoustic waves extracted from experimental data has revealed that the acoustic modes are intermittently switching from standing to clockwise or counter-clockwise spinning waves. ${ }^{64}$ Similar results were also found on academic experiments ${ }^{38}$ and large Eddy simulations of complete annular combustors. ${ }^{12,14,46,77}$

Noiray and Schuermans ${ }^{64}$ demonstrated that a deterministic approach cannot properly describe the actual modal dynamics encountered in turbulent gas turbines. Such a behavior can be explained 
by a high turbulence level, which randomly affects the mode structure, preventing a stationary limit cycle to settle in the annular combustion chamber. This calls for the introduction of stochastic forcing produced by the highly turbulent reactive flow for combustion instabilities. ${ }^{91-95}$

To do so, uncorrelated white noises $\zeta_{A}$ and $\zeta_{B}$ of intensity $\Gamma / 2 \omega_{m}^{2}$ have been added to the dynamical system established in Eq. (18). ${ }^{64,95}$ These coupled stochastic differential equations can be recast using the potential $\mathcal{V}(\mathcal{A}, \mathcal{B})$,

$$
\left\{\begin{array}{l}
\frac{d \mathcal{A}}{d t} \simeq-\frac{\partial \mathcal{V}}{\partial \mathcal{A}}+\zeta_{A} \\
\frac{d \mathcal{B}}{d t} \simeq-\frac{\partial \mathcal{V}}{\partial \mathcal{B}}+\zeta_{B}
\end{array},\right.
$$

where

$$
\begin{aligned}
& \mathcal{V}(\mathcal{A}, \mathcal{B})=-\frac{n_{0}}{4}\left[1+\frac{C_{2 m}}{2}\right] \mathcal{A}^{2}-\frac{n_{0}}{4}\left[1-\frac{C_{2 m}}{2}\right] \mathcal{B}^{2} \\
& +\frac{9 \kappa}{128}\left(\mathcal{A}^{4}+\mathcal{B}^{4}+\frac{2}{3} \mathcal{A}^{2} \mathcal{B}^{2}\right)-\frac{\Gamma}{4 \omega_{m}^{2}} \ln (\mathcal{A B}) .
\end{aligned}
$$

This equation and its associated Fokker-Planck equation provide the stationary bi-variate probability density function of the mode structure given by

$$
P(\mathcal{A}, \mathcal{B})=\frac{1}{\mathcal{N}} \exp \left(-\frac{4 \omega_{m}^{2}}{\Gamma} \mathcal{V}(\mathcal{A}, \mathcal{B})\right)
$$

where $\mathcal{N}$ is a normalization constant.

The coefficients of this model $\left(n_{0}, \kappa, C_{2 m}, \Gamma\right.$, and $\left.\omega_{m}\right)$ can then be identified using real gas turbine data to provide bi-variate density functions of the modal amplitudes. Figure 14 shows examples of joint PDFs obtained by Noiray and Schuermans ${ }^{64}$ where the noise intensity $\Gamma$ and the asymmetry level $C_{2 m}$ are varied. Note that when the turbulence intensity $\Gamma$ goes to zero, the joint PDF (Eq. (25)) goes to a dirac function, i.e., to a deterministic solution which depends only on the "splitting strength" $C_{2 m}$ ((c) in Fig. 14). However, when $\Gamma$ increases ((a) and (b) in Fig. 14), a large variety of mixed mode structures appears, as observed experimentally $38,39,82$ and numerically. ${ }^{46}$

\section{E. Quantification of uncertainties}

Section III B 2 has shown that symmetry breaking due to the introduction of damping devices or non-linear flame responses plays a crucial role in thermoacoustics, controlling both the stability and the dynamics of azimuthal acoustic modes. Section III D has shown that random turbulent forcing also affected the observed mode nature, leading to random changes over long times. This section focuses on another issue: the undesired introduction of symmetry breaking, for example, by manufacturing tolerances of burners ${ }^{96}$ which lead to small variations from one burner to its neighbour. Most gas turbines utilize injectors based on swirling flows. Swirling flows are very sensitive to small geometrical changes: this can lead to large effects on the flame response and therefore on the azimuthal mode stability. The goal is therefore to assess the effect of such uncertainties on the stability of acoustic modes.

In all previous approaches, the output of acoustic tools is typically a deterministic stability criterion (e.g., Eq. (19)) or a map of the thermo-acoustic modes in the complex plane (see the black symbols in Fig. 15). In this view, each mode is actually either stable $(\operatorname{Im}(f)<0)$ or unstable $(\operatorname{Im}(f)>0)$, depending on the input parameters of the thermoacoustic analysis.

The stability analysis is more complex when the input parameters are uncertain. For example, the speed of sound, the boundary impedances, and the flame forcing are very sensitive to multiple physical parameters such as the flow regime, manufacturing tolerances, fuel changes, turbulence, etc., which are partly unknown. ${ }^{27,56,97}$ As a consequence, the deterministic view (one single growth rate and frequency for each mode) must be replaced by an uncertain region of the complex plane (Fig. 15) where the mode frequency can be found depending on the uncertain input parameters. When this region is known, it can be conveniently characterized by a risk factor, i.e., the probability 


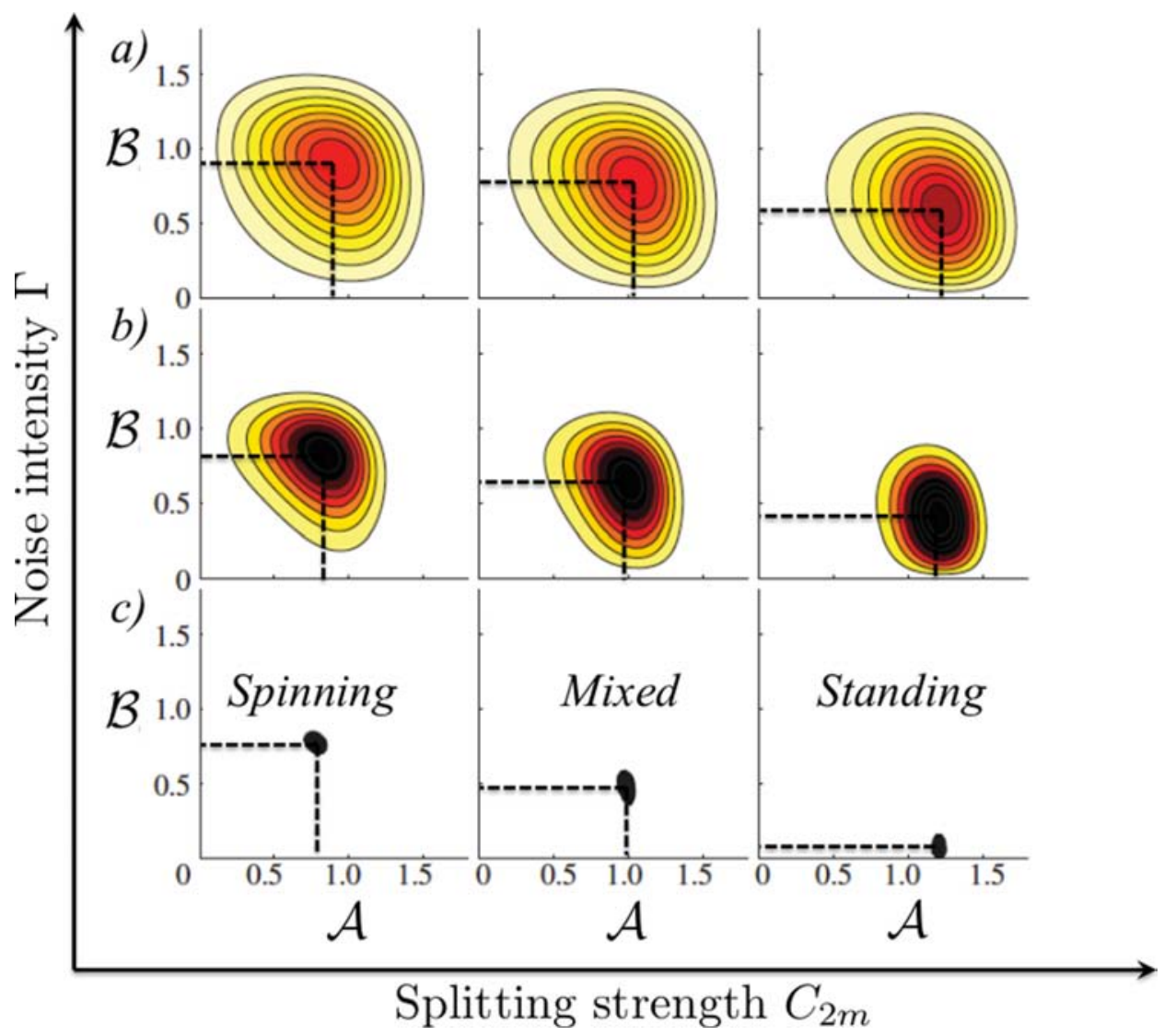

FIG. 14. Joint probability density functions of the modal amplitudes $\mathcal{A}$ and $\mathcal{B}$ using Fokker-Planck solution (25) when both the noise intensity $\Gamma$ and the asymmetry level $C_{2 m}$ are varied. The mode categories (spinning, mixed, or standing) can be determined only at low turbulence intensity $\Gamma$. In this case, the mode type is controlled by the asymmetry, measured here by the coefficient $C_{2 m}$. Reprinted with permission from Noiray and Schuermans, "On the dynamic nature of azimuthal thermoacoustic modes in annular gas turbine combustion chambers," Proc. R. Soc. A 469, 1471-2946 (2013). Copyright 2013 Royal Society.

of a mode to be unstable,

$$
R F(\%)=100 \int_{0}^{\infty} \operatorname{PDF}\left(f_{I m}\right) d f_{I m},
$$

where $f_{I m}$ is the imaginary part of the complex frequency $f$, and $\operatorname{PDF}\left(f_{I m}\right)$ is the probability density function of the growth rate $f_{I m}$ (an example is given in Fig. 16, right), knowing that by

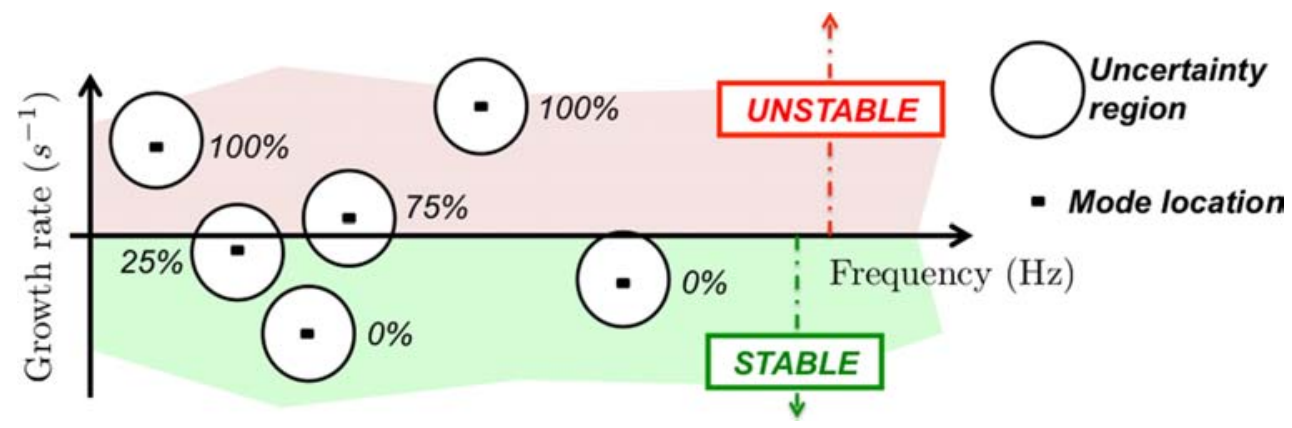

FIG. 15. Location in the frequency plane of the first six thermo-acoustic modes in a typical combustor without uncertainties (single point, black symbols) and with uncertainties (each mode belongs to an admissible region of the frequency plane with an associated risk factor). 

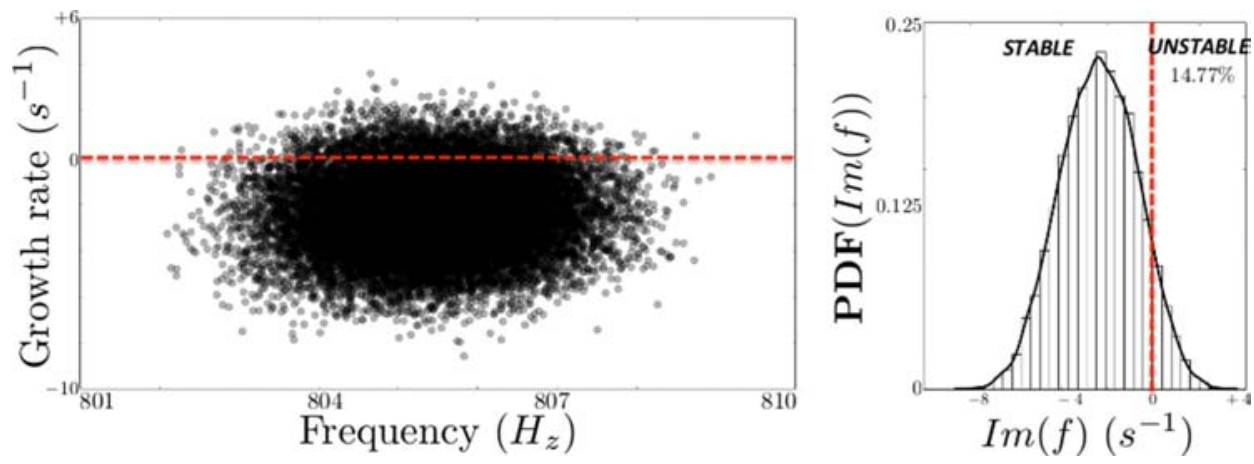

FIG. 16. Monte Carlo analysis using 10000 ATACAMAC simulations for the first weakly coupled azimuthal chamber mode. Each point on the stability map (left) corresponds to one ATACAMAC simulation. This method allows a robust estimation of PDFs and risk factors (right).

definition $\int_{-\infty}^{\infty} \operatorname{PDF}\left(f_{I m}\right) d f_{I m}=1$. This novel notion renews the classical bi-modal stable/unstable view in thermo-acoustics, but it requires the computation of the complete PDF of the growth rate to obtain the risk factor. Such an approach has been performed using $3 D$ Helmholtz solvers for a single swirled burner where two parameters were assumed uncertain: the gain $n$ and the time-delay $\tau$ of the FTF. $^{98}$

The probabilistic estimation of the risk factor is much more complicated when dealing with realistic annular combustors, since the number of uncertain parameters increases drastically. Typically, the number of uncertain parameters may reach several tens since the gains $n_{i}$ and time-delays $\tau_{i}$ of each flame $(i=1-N)$ are highly sensitive to manufacturing tolerances. Consider, for example, a perfectly symmetric PBC configuration ((d) in Fig. 6) containing $N=19$ burners: assuming that the only uncertain input data are the FTFs parameters $n_{i}$ and $\tau_{i}$ leads to 38 uncertain variables. Studying the stability map with 38 uncertain parameters is out of reach of LES but it can be achieved efficiently with analytical and semi-analytical models. ${ }^{96}$ Here, ATACAMAC is used to solve numerically the dispersion relation (Eq. (4)). 10000 simulations of the first $(m=1)$ weakly coupled chamber mode of the configuration are performed (Fig. 16). The operating point corresponds to weakly coupled modes $\left(n_{0}=0.5\right.$ and $\tau_{0}=0.635 \mathrm{~ms}$ ) for which each flame is uncertain (uniform distribution centered at $\left(n_{0}, \tau_{0}\right)$ with standard deviations $\sigma_{n}=0.1 n_{0}$ and $\tau=0.05 \tau_{0}$ ). Therefore, the PDF functions for the gain $n$ and the time-delay $\tau$ are the same for all the 19 flames, but random values $\left(n_{i}, \tau_{i}\right)$ chosen according to this probability density function are different for each flame: the flames are considered as statistically independent, as expected for uncertainties due to manufacturing tolerances, for instance. For this mode, the risk factor is estimated to $15 \%$, while the deterministic growth rate was estimated at $-2.33 \mathrm{~s}^{-1}$, i.e., a stable mode: the mode needs to be controlled despite the deterministic prediction.

Compared with Section III D where a uniform noise was added to the non-symmetric configuration, here a non-uniform uncertainty distribution (i.e., each parameter, $n_{i}$ and $\tau_{i}$, is chosen independently, so that the discrete heat release distribution is not axisymmetric) is imposed in a perfectly symmetric combustor. Note that the mean (in a statistical sense) heat release distribution is symmetric, and only the stochastic perturbations around this state are non-symmetric so that symmetry breaking due to non-uniformity between burners should appear. It is not visible in Fig. 16 since the numerical predictions cannot be identified as $f_{m}^{+}$and $f_{m}^{-}$in Eq. (9). In other words, compared with analytical methods, semi-analytical tools allow accurate computations of both weakly and strongly coupled modes, but fail in the understanding of the underlying physics (here the splitting effect). To retrieve such physical interpretation and extract properly the splitting effect due to uncertainties in the numerical database, an EM algorithm (Estimation-Minimization, Dempster, and Laird ${ }^{99}$ ) is used: analytical and numerical tools are thus combined. It is based on a two-step regression using the analytical model obtained in Eq. (9):

- Estimation: A linear surrogate model $\widetilde{f_{I m}^{k}}$ of the growth rate $f_{I m}^{k}$ of the $k$ th sample is fit on the Monte Carlo data, based on the analytical coupling $\Sigma_{0}^{k}$ and splitting strength $\mathcal{S}_{0}^{k}$ (Eq. (9)), as 


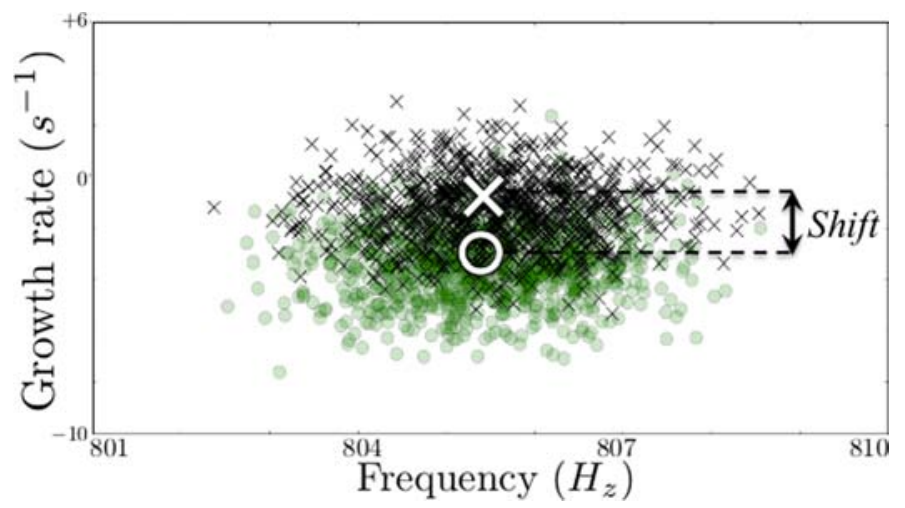

FIG. 17. The two components of the first $(m=1)$ azimuthal mode $\left(\times\right.$ when $Z^{k}=+1$ and $\circ$ when $\left.Z^{k}=-1\right)$ from 1000 ATACAMAC simulations obtained by the variable $Z$ in the EM-algorithm. The large white cross and circle display the mean location of each component and their respective shift.

well as an additional variable $Z^{k}$ with values in $\{+1,-1\}$,

$$
\widetilde{f_{I m}^{k}}=a_{0} \Sigma_{0}^{k}+a_{1} Z^{k} \mathcal{S}_{0}^{k}
$$

At this step, an estimation of the coefficients $a_{0}$ and $a_{1}$ is obtained, assuming values for $Z^{k}$. A random initialization of this variable $Z^{k}$ is therefore required before this step, since it is unknown: the objective of such an EM-algorithm is to exemplify the splitting effect due to the uncertainties, by segregating the two azimuthal components $f^{+}$and $f^{-}$(Eq. (9)), i.e., to find the values of the variable $Z^{k}= \pm 1$. Note that this variable $Z^{k}$ appears only in the splitting term proportional to $\mathcal{S}_{0}^{k}$, and therefore segregates the two azimuthal components. If the analytical coupling $\Sigma_{0}^{k}$ and splitting strength $\mathcal{S}_{0}^{k}$ are not known, a numerical method to approximate them from the Monte Carlo database, called active subspace, ${ }^{100}$ can be performed before applying this step. ${ }^{46,96,101}$

- Minimization: Then, the previous model can be reused to evaluate a new variable $Z^{k}$, thanks to a minimization procedure: for each sample $k$, the variable $Z^{k}$ is changed into $-Z^{k}$. If this new value yields a better estimation of the actual growth rate $f_{I m}^{k}$, this value is retained. A new estimation step can then be performed, leading to an iterative method to obtain both a surrogate model (coefficients $a_{0}$ and $a_{1}$ ) and the segregation between the two components of the azimuthal mode (given by $Z$ ). The convergence of such an iterative algorithm is however not ensured and therefore should be monitored carefully.

Results of the EM algorithm for the first $(m=1)$ weakly coupled case are shown in Fig. 17. The two splitted components $(\times$ and $\circ$ ) are determined by the variable $Z$ : the two clouds of points $(X$ and $\circ)$ are shifted in the growth rate direction (the mean location of each cloud is displayed by a large white cross and circle in Fig. 17). A strong splitting effect occurs and destabilizes the configuration. This symmetry breaking is necessarily due to uncertainties, since they are the only source of non-uniformity. It proves that non-uniform uncertainties on the flame model leads to symmetry breaking effects and consequently to a potential destabilization of the configuration.

\section{CONCLUSION}

Compared with longitudinal modes which have been investigated for decades, azimuthal modes present in annular combustors constitute a specific case where combustion instabilities still raise multiple fundamental questions. Mechanisms leading to azimuthal instabilities are more complex than those encountered in longitudinal configurations because of the multiple burners and their potential interactions. Because of their complexity and costs, high fidelity simulations or experimental annular rigs are prohibitive to study azimuthal modes in a systematic manner. Consequently, for such circumferential acoustic modes, theoretical analysis have progressed recently to analyze 
underlying phenomena controlling the stability, nature, and dynamics of these peculiar acoustic modes. First, the truncated Galerkin method and network models are presented with their pros and cons in both the temporal and frequency framework. Then, key features of such acoustic modes are unveiled, focusing on their specificities to classify them into several categories which control their stability, nature, and dynamics (coupled vs. decoupled, degenerate vs. non-degenerate, linear vs. non-linear, spinning vs. standing modes, and finally deterministic vs. stochastic approaches). This review reveals that many aspects of azimuthal modes involve symmetry breaking: the rotational symmetry breaking appears as an essential element of research for circumferential acoustic modes, which can be discovered only by theory. These theoretical findings can then be investigated in details by dedicated experiments or high fidelity simulations. In particular, choked experimental rigs to study pure azimuthal modes are still missing. Moreover, while recent UQ and sensitivity analysis have been introduced, the knowledge of their inputs (e.g., uncertainties on the flame model parameters) still request a computational and experimental effort. Finally, to predict accurately unstable modes in annular combustors and stabilize them if they appear, dedicated studies on acoustic damping and innovative control techniques are necessary. In this context, analytical analysis remains essential to guide future simulations and experiments.

\section{ACKNOWLEDGMENTS}

This work was granted access to the high-performance computing resources of CINES by Grand Equipement National de Calcul Intensif. The support of the European commission, through the ERC advanced grant INTECOCIS GA 319067, is acknowledged.

${ }^{1}$ F. E. C. Culick and P. Kuentzmann, Unsteady Motions in Combustion Chambers for Propulsion Systems (NATO Research and Technology Organization, 2006).

2 T. Lieuwen and V. Yang, Combustion Instabilities in Gas Turbines Engines, Progress in Astronautics and Aeronautics Vol. 210 (AIAA, 2005)

${ }^{3}$ C. Goy, S. James, and S. Rea, "Monitoring combustion instabilities: E.ON UK's experience," in Combustion Instabilities in Gas Turbines Engines (AIAA, 2005), Chap. 8, pp. 163-175.

${ }^{4}$ T. Poinsot and D. Veynante, Theoretical and Numerical Combustion, 3rd ed. (2011), www.cerfacs.fr/elearning.

${ }^{5}$ S. Candel, "Combustion dynamics and control," Proc. Combust. Inst. 29, 1-28 (2002).

${ }^{6}$ D. J. Harrje and F. H. Reardon, "Liquid propellant rocket instability," Technical Report SP-194, NASA, 1972.

${ }^{7}$ V. Yang and W. Anderson, Liquid Rocket Engine Combustion Instability, Progress in Astronautics and Aeronautics Vol. 169 (AIAA, 1995)

8 T. Poinsot, A. Trouvé, D. Veynante, S. Candel, and E. Esposito, "Vortex driven acoustically coupled combustion instabilities," J. Fluid Mech. 177, 265-292 (1987).

${ }^{9}$ J. G. Lee and D. Santavicca, "Experimental diagnostics for the study of combustion instabilities in lean premixed combustors," J. Propul. Power 19, 735-750 (2003)

${ }^{10}$ D. S. Lee and T. J. Anderson, "Measurements of fuel/air-acoustic coupling in lean premixed combustion systems," AIAA Paper 99-0450, 1999.

${ }^{11}$ K. K. Venkataraman, L. H. Preston, S. D. W. B. Lee, J. Lee, and D. Santavicca, "Mechanism of combustion instability in a lean premixed dump combustor,” J. Propul. Power 15, 909-918 (1999).

12 P. Wolf, G. Staffelbach, A. Roux, L. Gicquel, T. Poinsot, and V. Moureau, "Massively parallel LES of azimuthal thermoacoustic instabilities in annular gas turbines," C. R. Acad. Sci. Méc. 337, 385-394 (2009).

${ }^{13}$ G. Staffelbach, L. Gicquel, G. Boudier, and T. Poinsot, "Large eddy simulation of self-excited azimuthal modes in annular combustors," Proc. Combust. Inst. 32, 2909-2916 (2009).

${ }^{14}$ P. Wolf, G. Staffelbach, R. Balakrishnan, A. Roux, and T. Poinsot, "Azimuthal instabilities in annular combustion chambers," in Proceedings of the Summer Program (Center for Turbulence Research, Stanford University, 2010), pp. 259-269.

15 A. P. Dowling, "The calculation of thermoacoustic oscillations," J. Sound Vib. 180, 557-581 (1995).

16 A. P. Dowling, "Nonlinear self-excited oscillations of a ducted flame," J. Fluid Mech. 346, 271-290 (1997).

${ }^{17}$ J. Kopitz, A. Huber, T. Sattelmayer, and W. Polifke, "Thermoacoustic stability analysis of an annular combustion chamber with acoustic low order modeling and validation against experiment," ASME Paper 2005-GT-68797, 2005.

${ }^{18}$ S. Evesque and W. Polifke, "Low-order acoustic modelling for annular combustors: Validation and inclusion of modal coupling," ASME Paper 2002-GT-30064, 2002.

${ }^{19}$ F. Nicoud, L. Benoit, C. Sensiau, and T. Poinsot, "Acoustic modes in combustors with complex impedances and multidimensional active flames," AIAA J. 45, 426-441 (2007).

${ }^{20}$ S. Candel, "Combustion instabilities coupled by pressure waves and their active control," Proc. Combust. Inst. 24, 1277-1296 (1992).

${ }^{21}$ D. G. Crighton, A. P. Dowling, J. E. F. Williams, M. Heckl, and F. Leppington, Modern Methods in Analytical Acoustics, Lecture Notes (Springer-Verlag, New York, 1992).

22 J. O'Connor and T. Lieuwen, “Transverse combustion instabilities: Acoustic, fluid mechanics and flame processes," Prog. Energy Combust. Sci. 49, 1-39 (2014) 
${ }^{23}$ J. Blimbaum, M. Zanchetta, T. Akin, V. Acharya, J. O'Connor, D. Noble, and T. Lieuwen, "Transverse to longitudinal acoustic coupling processes in annular combustion chambers," Int. J. Spray Combust. Dyn. 4, 275-298 (2012).

${ }^{24}$ F. Lespinasse, F. Baillot, and T. Boushaki, "Response of V-flames placed in an HF transverse acoustic field from a velocity to pressure antinode," C. R. Acad. Sci. Méc. 341, 110-120 (2013)

${ }^{25}$ J. O'Connor and T. Lieuwen, "Recirculation zone dynamics of a transversely excited swirl flow and flame," Phys. Fluids 24, 075107 (2012)

${ }^{26} \mathrm{~J}$. O'Connor and T. Lieuwen, "Influence of transverse acoustic modal structure on the forced response of a swirling nozzle flow," ASME Paper 2012-GT-70053, 2012.

${ }^{27}$ M. Bauerheim, G. Staffelbach, N. Worth, J. Dawson, L. Gicquel, and T. Poinsot, "Sensitivity of les-based harmonic flame response model for turbulent swirled flames and impact on the stability of azimuthal modes," Proc. Combust. Inst. $\mathbf{3 5}$, 3355-3363 (2014).

${ }^{28}$ G. Singla, N. Noiray, and B. Schuermans, "Combustion dynamics validation of an annular reheat combustor," ASME Paper 2012-68684, 2012

${ }^{29}$ M. Zellhuber, J. Schwing, B. Schuermans, T. Sattelmayer, and W. Polifke, "Experimental and numerical investigation of thermoacoustic sources related to high-frequency instabilities," Int. J. Spray Combust. Dyn. 6, 1-34 (2014).

${ }^{30}$ M. Bauerheim, J. Parmentier, P. Salas, F. Nicoud, and T. Poinsot, "An analytical model for azimuthal thermoacoustic modes in an annular chamber fed by an annular plenum," Combust. Flame 161, 1374-1389 (2014).

31 T. Schuller, D. Durox, P. Palies, and S. Candel, "Acoustic decoupling of longitudinal modes in generic combustion systems," Combust. Flame 159, 1921-1931 (2012).

32 J.-F. Bourgouin, "Dynamique de flamme dans les foyeres annulaires comportant des injecteurs multiples," Ph.D. thesis, Ecole Centrale de Paris, EM2C, 2014.

${ }^{33}$ S. Camporeale, B. Fortunato, and G. Campa, "A finite element method for three-dimensional analysis of thermo-acoustic combustion instability," J. Eng. Gas Turbines Power 133, 011506 (2011).

${ }^{34}$ W. Krebs, P. Flohr, B. Prade, and S. Hoffmann, "Thermoacoustic stability chart for high-intensity gas turbine combustion systems," Combust. Sci. Technol. 174, 99-128 (2002).

35 B. Schuermans, C. Paschereit, and P. Monkewitz, "Non-linear combustion instabilities in annular gas-turbine combustors," AIAA Paper 2006-0549, 2006.

${ }^{36}$ S. Evesque, W. Polifke, and C. Pankiewitz, "Spinning and azimuthally standing acoustic modes in annular combustors," in AIAA Paper 2003-3182, 2003.

${ }^{37}$ R. Perrin and T. Charnley, "Group theory and the bell," J. Sound Vib. 31, 411-418 (1973).

${ }^{38}$ N. Worth and J. Dawson, "Self-excited circumferential instabilities in a model annular gas turbine combustor: Global flame dynamics," Proc. Combust. Inst. 34, 3127-3134 (2013).

${ }^{39}$ N. Worth and J. Dawson, "Modal dynamics of self-excited azimuthal instabilities in an annular combustion chamber," Combust. Flame 160, 2476-2489 (2013).

${ }^{40}$ N. Noiray, M. Bothien, and B. Schuermans, "Investigation of azimuthal staging concepts in annular gas turbines," Combust. Theory Modell. 15, 585-606 (2011).

${ }^{41}$ C. Sensiau, "Simulations numériques des instabilités thermoacoustiques dans les chambres de combustion aéronautiques TH/CFD/08/127," Ph.D. thesis, Université de Montpellier II, Institut de Mathématiques et de Modélisation de Montpellier, France, 2008

${ }^{42}$ C. Sensiau, F. Nicoud, and T. Poinsot, "A tool to study azimuthal and spinning modes in annular combustors," Int. J. Aeroacoustics 8, 57-68 (2009).

${ }^{43}$ J.-F. Bourgouin, D. Durox, J. Moeck, T. Schuller, and S. Candel, "A new pattern of instability observed in an annular combustor: The slanted mode," Proc. Combust. Inst. 35, 3237-3244 (2014).

44 J.-F. Bourgouin, D. Durox, J. Moeck, T. Schuller, and S. Candel, "Self-sustained instabilities in an annular combustor coupled by azimuthal and longitudinal acoustic modes," ASME Paper 2013-GT-95010, 2013.

${ }^{45}$ C. Fureby, "LES of a multi-burner annular gas turbine combustor," Flow, Turbul. Combust. 84, 543-564 (2010).

${ }^{46} \mathrm{M}$. Bauerheim, "Theoretical and numerical study of symmetry breaking effects on azimuthal thermoacoustic modes in annular combustors," Ph.D. thesis, INP Toulouse \& Cerfacs, 2014.

${ }^{47}$ F. A. Williams, Combustion Theory (Benjamin Cummings, Menlo Park, CA, 1985)

${ }^{48}$ P. Clavin and G. Joulin, "Premixed flames in large scale and high intensity turbulent flow," J. Phys. Lett. 44, L1-L12 (1983).

${ }^{49}$ Lord Rayleigh, The Theory of Sound (Macmillan, 1894), reprinted by (Dover, New York, 1945).

${ }^{50}$ G. Searby, "Prediction of the efficiency of acoustic damping cavities," J. Propul. Power 24, 516-523 (2008).

${ }^{51}$ L. Crocco, "Research on combustion instability in liquid propellant rockets," Proc. Combust. Inst. 12, 85-99 (1969).

${ }^{52}$ N. Noiray, D. Durox, T. Schuller, and S. Candel, "A unified framework for nonlinear combustion instability analysis based on the flame describing function," J. Fluid Mech. 615, 139-167 (2008).

${ }^{53}$ T. Sattelmayer, "Influence of the combustor aerodynamics on combustion instabilities from equivalence ratio fluctuations," J. Eng. Gas Turbines Power 125, 11-19 (2003).

${ }^{54}$ W. Polifke, A. Poncet, C. O. Paschereit, and K. Doebbeling, "Reconstruction of acoustic transfer matrices by instationnary computational fluid dynamics," J. Sound Vib. 245, 483-510 (2001)

${ }^{55}$ F. Nicoud and K. Wieczorek, "About the zero Mach number assumption in the calculation of thermoacoustic instabilitie," Int. J. Spray Combust. Dyn. 1, 67-112 (2009).

${ }^{56} \mathrm{~K}$. Kedia, H. Altay, and A. Ghoniem, "Impact of flame-wall interaction on premixed flame dynamics and transfer function characteristics," Proc. Combust. Inst. 33, 1113-1120 (2011).

${ }^{57}$ E. Motheau, L. Selle, and F. Nicoud, "Accounting for convective effects in zero-Mach-number thermoacoustic models," J Sound Vib. 333, 246-262 (2014).

${ }^{58}$ D. Mejia, L. Selle, R. Bazile, and T. Poinsot, "Wall-temperature effects on flame response to acoustic oscillations," Proc. Combust. Inst. 35, 3201-3208 (2014).

${ }^{59}$ S. Stow and A. Dowling, "Low-order modelling of thermoacoustic limit cycles," ASME Paper 2004-GT-54245, 2004. 
${ }^{60}$ M. Juniper, “Triggering in the horizontal Rijke tube: Non-normality, transient growth and bypass transition," J. Fluid Mech. 667, 272-308 (2011).

${ }^{61}$ N. Noiray, M. Bothien, and B. Schuermans, "Investigation of azimuthal staging concepts in annular gas turbines," Combust. Theory Modell. 15, 585-606 (2011).

${ }^{62}$ G. Ghirardo and M. Juniper, "Azimuthal instabilities in annular combustors: Standing and spinning modes," Proc. R. Soc. A 469, 20130232 (2013).

${ }^{63}$ G. Ghirardo, M. Juniper, and J. Moeck, "Stability criteria for standing and spinning waves in annular combustors," ASME Paper GT2015-43127, 2015

${ }^{64}$ N. Noiray and B. Schuermans, "On the dynamic nature of azimuthal thermoacoustic modes in annular gas turbine combustion chambers," Proc. R. Soc. A 469, 1471-2946 (2013).

${ }^{65}$ G. Gelbert, J. Moeck, C. Paschereit, and R. King, "Feedback control of unstable thermoacoustic modes in an annular Rijke tube," Control Eng. Pract. 20, 770-782 (2012).

${ }^{66}$ J.-F. Bourgouin, D. Durox, J. Moeck, T. Schuller, and S. Candel, "Characterization and modeling of a spinning thermoacoustic instability in an annular combustor equipped with multiple matrix injectors," J. Eng. Gas Turbines Power 137, 021503 (2014).

${ }^{67}$ U. Krüger, J. Hüren, S. Hoffmann, W. Krebs, P. Flohr, and D. Bohn, "Prediction and measurement of thermoacoustic improvements in gas turbines with annular combustion systems," J. Eng. Gas Turbines Power 123, 557-566 (2000)

${ }^{68}$ S. R. Stow and A. P. Dowling, "Thermoacoustic oscillations in an annular combustor," ASME Paper 2001-GT-0037, 2001

${ }^{69}$ S. R. Stow and A. P. Dowling, "Modelling of circumferential modal coupling due to helmholtz resonators," ASME Paper 2003-GT-38168, 2003.

${ }^{70}$ J. Parmentier, P. Salas, P. Wolf, G. Staffelbach, F. Nicoud, and T. Poinsot, "A simple analytical model to study and control azimuthal instabilities in annular combustion chamber," Combust. Flame 159, 2374-2387 (2012).

${ }^{71}$ M. Bauerheim, M. Cazalens, and T. Poinsot, "A theoretical study of mean azimuthal flow and asymmetry effects on thermo-acoustic modes in annular combustors," Proc. Combust. Inst. 35, 3219-3227 (2014).

72 M. Bauerheim, P. Salas, F. Nicoud, and T. Poinsot, "Symmetry breaking of azimuthal thermoacoustic modes in annular cavities: A theoretical study," J. Fluid Mech. 760, 431-465 (2014).

${ }^{73}$ M. Bauerheim, F. Nicoud, and T. Poinsot, "Theoretical analysis of the mass balance equation through a flame at zero and non-zero Mach numbers," Combust. Flame 162, 60-67 (2014).

${ }^{74}$ L. Crocco, "Theoretical studies on liquid propellant rocket instability," Symp. (Int.) Combust. 10, 1101-1128 (1965).

${ }^{75}$ K. Balasubramanian and R. Sujith, "Thermoacoustic instability in a Rijke tube: Non-normality and non-linearity,” Phys. Fluids 20, 044103 (2008).

${ }^{76}$ C. Silva, F. Nicoud, T. Schuller, D. Durox, and S. Candel, "Combining a helmholtz solver with the flame describing function to assess combustion instability in a premixed swirled combustor," Combust. Flame 160, 1743-1754 (2013).

${ }^{77}$ P. Wolf, G. Staffelbach, L. Gicquel, J. Muller, and T. Pionsot, "Acoustic and large eddy simulation studies of azimuthal modes in annular combustion chambers," Combust. Flame 159, 3398-3413 (2012).

${ }^{78}$ C. Pankiewitz and T. Sattelmayer, "Time domain simulation of combustion instabilities in annular combustors," J. Eng. Gas Turbines Power 125, 677-685 (2003)

${ }^{79} \mathrm{G}$. Campa and S. Camporeale, "Influence of nonlinear effects on the limit cycle in a combustion chamber equipped with helmholtz resonators," ASME Paper 2014-GT-25228, 2014.

${ }^{80} \mathrm{G}$. Mensah and J. Moeck, "Efficient computation of thermoacoustic modes in annular combustion chambers based on bloch-wave theory," ASME Paper GT2015-43476, 2015.

${ }^{81}$ M. Bauerheim, T. Jaravel, L. Esclapez, R. Riber, L. Gicquel, B. Cuenot, S. Bourgois, M. Rullaux, and M. Cazalens, "Multiphase flow les study of the fuel split effects on combustion instabilities in an ultra low-nox annular combustor," ASME Paper GT2015-44139, 2015

82 J. Dawson and N. Worth, "The effect of baffles on self-excited azimuthal modes in an annular combustor," Proc. Combust. Inst. 35, 3283-3290 (2014).

${ }^{83}$ P. Berenbrink and S. Hoffmann, "Suppression of dynamic combustion instabilities by passive and active means," ASME Paper 2001-GT-42, 2001.

${ }^{84}$ J. Moeck, M. Paul, and C. Paschereit, “Thermoacoustic instabilities in an annular flat Rijke tube," ASME Paper 2010-GT23577, 2010.

${ }^{85}$ E. Lavely, "Theoretical investigations in helioseismology," Ph.D. thesis, Columbia University, 1983.

${ }^{86}$ D. Cummings and D. Blackburn, "Oscillations of magnetically levitated aspherical droplets," J. Fluid Mech. 224, 395-416 (1991).

${ }^{87}$ F. Busse, "Oscillations of a rotating liquid drop," J. Fluid Mech. 142, 1-8 (1984).

${ }^{88}$ J. A. Creighton, "Splitting of degenerate vibrational modes due to symmetry perturbations in tetrahedral M4 and octahedral M6 clusters," Inorg. Chem. 21, 1-4 (1982).

${ }^{89}$ A. Davey and H. Salwen, "On the stability of flow in an elliptic pipe which is nearly circular," J. Fluid Mech. 281, 357-369 (1994).

${ }^{90} \mathrm{P}$. Salas, "Aspects numériques et physiques des instabilités thermoacoustiques dans les chambres de combustion annulaires," Ph.D. thesis, Université Bordeaux I, 2013.

${ }^{91}$ P. Clavin, J. Kim, and F. Williams, "Turbulence-induced noise effects on high-frequency combustion instabilities," Combust. Sci. Technol. 96, 61-84 (1994).

92 T. Lieuwen, "Statistical characteristics of pressure oscillations in a premixed combustor," J. Sound Vib. 260, 3-17 (2003).

${ }^{93}$ T. Lieuwen and A. Banaszuk, "Background noise effects on combustor stability," J. Propul. Power 21, 25-31 (2005).

${ }^{94}$ I. Waugh, M. Geuss, and M. Juniper, "Triggering, bypass transition and the effect of noise on a linearly stable thermoacoustic system," Proc. Combust. Inst. 33, 2945-2952 (2011).

${ }^{95}$ N. Noiray and B. Schuermans, "Deterministic quantities characterizing noise driven Hopf bifurcations in gas turbine combustor," Int. J. Nonlinear Mech. 50, 152-163 (2013). 
${ }^{96}$ M. Bauerheim, A. Ndiaye, P. Constantine, G. Iaccarino, S. Moreau, and F. Nicoud, "Uncertainty quantification of thermo-acoustic instabilities in annular combustors," in Proceedings of the Summer Program (Center for Turbulence Research, Stanford University, 2014), pp. 209-218.

${ }^{97}$ F. Duchaine, F. Boudy, D. Durox, and T. Poinsot, "Sensitivity analysis of transfer functions of laminar flames," Combust. Flame 158, 2384-2394 (2011).

${ }^{98}$ A. Ndiaye, M. Bauerheim, S. Moreau, and F. Nicoud, "Uncertainty quantification of thermo-acoustic instabilities in a swirled stabilized combustor," ASME Paper 2015-GT-44133, 2015.

${ }^{99}$ A. Dempster and N. Laird, "Maximum likelihood from incomplete data via the em algorithm," J. R. Stat. Soc., Ser. B 39, 1-38 (1977).

100 P. G. Constantine, E. Dow, and Q. Wang, "Active subspace methods in theory and pratice: Applications to kriging surfaces," SIAM J. Sci. Comput. 36, 1500-1524 (2014).

${ }^{101}$ M. Juniper, L. Magri, M. Bauerheim, and F. Nicoud, "Applications of adjoint methods in thermoacoustics," in Proceedings of the Summer Program (Center for Turbulence Research, Stanford University, 2015), pp. 189-198. 